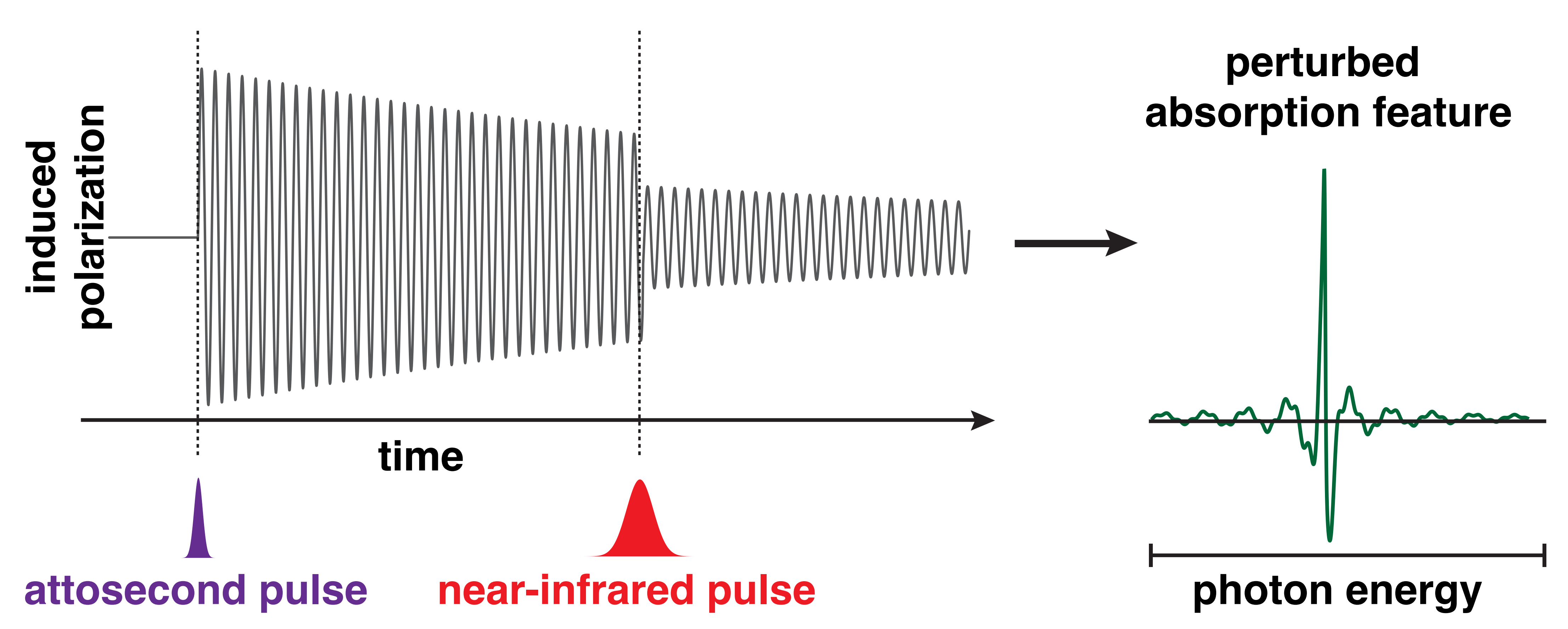




\title{
Probing ultrafast dynamics with attosecond transient absorption
}

\author{
Annelise R. Beck ${ }^{1 *}$, Daniel M. Neumark ${ }^{1}$, Stephen R. Leone ${ }^{1,2^{*}}$ \\ ${ }^{1}$ Department of Chemistry, University of California Berkeley, and Chemical Sciences Division, Lawrence Berkeley \\ National Laboratory, Berkeley, California, 94720, USA \\ ${ }^{2}$ Department of Physics, University of California Berkeley, Berkeley, California, 94720, USA \\ *Corresponding author. E-mail: arbeck@berkeley.edu (A.R.B.); srl@ berkeley.edu (S.R.L).
}

Keywords: attosecond spectroscopy, transient absorption, ultrafast dynamics

\begin{abstract}
:
The broad bandwidth of an isolated attosecond pulse excites a vast number of states simultaneously, and the corresponding absorption features can be monitored with exceptional temporal resolution. Novel transient absorption experiments in gases using isolated attosecond pulses are performed in two regimes, one in which the attosecond pulse is overlapped in time with a near-infrared (NIR) pulse and one in which the NIR pulse follows the attosecond pulse. In the latter regime, the attosecond pulse first interacts with a sample, then the observed absorption features are modified by a NIR pulse, which interacts with the sample well after the attosecond pulse has passed. In these experiments, which seem counterintuitive when compared to conventional transient absorption spectroscopy, the weak attosecond pulse induces a polarization of the medium, which is then perturbed by the time-delayed NIR pulse. Recent measurements demonstrate the rich variety of information that can be extracted in this regime.
\end{abstract}

\section{Introduction}

Electronic dynamics in atoms and molecules typically occur on a fewfemtosecond or sub-femtosecond timescale. These processes are too fast to be resolved by ordinary femtosecond optical laser pulses. However, in the past ten years, techniques to generate isolated pulses with durations of a few hundred attoseconds, or even shorter, have been established. These isolated attosecond pulses provide unprecedented opportunities to study ultrafast electron dynamics on a few-femtosecond timescale.

Isolated attosecond pulses are generated using high harmonic generation, in which a femtosecond laser pulse is upconverted to produce photon energies in the extreme ultraviolet (XUV) [1], in conjunction with optical gating techniques. The short duration of the pulse necessarily results in a broad and continuous spectrum, with bandwidths ranging from a few electron volts $(\mathrm{eV})$ to tens of $\mathrm{eV}$ and central photon energies typically between $10 \mathrm{eV}$ and $100 \mathrm{eV}$. These ultrashort pulses have been used in many successful experiments incorporating photoelectron and ion detection methods. For example, attosecond pulses have been used to measure the few-femtosecond timescale of Auger 
decay after core excitation of krypton [2] and to measure the time delay between the emission of an electron from two different atomic orbitals in neon [3].

The broad and continuous bandwidth of an isolated attosecond pulse, with photon energies in the extreme ultraviolet (XUV), is well suited to absorption measurements. The XUV photon energies can access highly excited states such as Rydberg states approaching the ionization limit or autoionizing states embedded in the ionization continuum. Electronic dynamics can be tracked in real time by monitoring the resulting changes in the absorption spectrum.

The majority of attosecond transient absorption experiments have been performed on rare gas atoms, which are relatively simple systems that still manifest complex behavior. However, the recent application in our group of attosecond transient absorption to observe the transfer of electrons from the valence band to conduction band in solid silicon [4] illustrates the generality of the technique. Attosecond transient absorption will soon be extended to study gas phase molecular systems, but the focus of this article is the experiments performed on rare gas atoms, in which a variety of novel phenomena have been identified.

Isolated attosecond pulses can be utilized in novel transient absorption experiments, in which the XUV attosecond pulse first passes through the sample, then a near-infrared (NIR) laser pulse follows at a time delay. It may seem counterintuitive that a change in the absorption spectrum of the XUV pulse can be observed when the NIR pulse follows the XUV pulse. This unconventional pulse order is possible because the XUV pulse induces a polarization of the medium. This polarization persists long after the XUV pulse passes through the sample, and it dephases on a timescale that depends on the lifetime of the states excited, which can range from tens of femtoseconds for a shortlived autoionizing state to nanoseconds for an atomic Rydberg level. The subsequent NIR pulse perturbs this polarization when it interacts with the sample. The macroscopic polarization is formed by a collection of oscillating dipole moments, which emit light. This emission is then dispersed in a spectrometer, essentially measuring the Fourier transform of the time-dependent signal. Absorption features are observed due to destructive interference between the emission from the excited sample and the transmitted broadband pulse. Because the induced polarization has been perturbed by the NIR pulse, the measured absorption features change. This is shown schematically in Figure 1. 
(a)

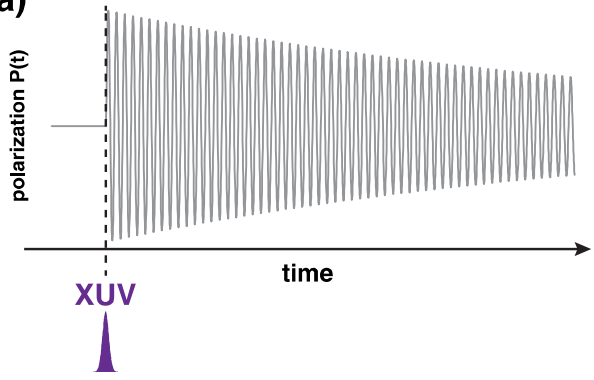

(b)

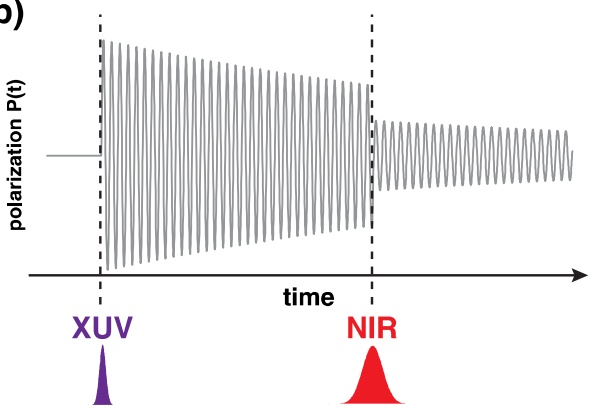

Transmitted spectrum
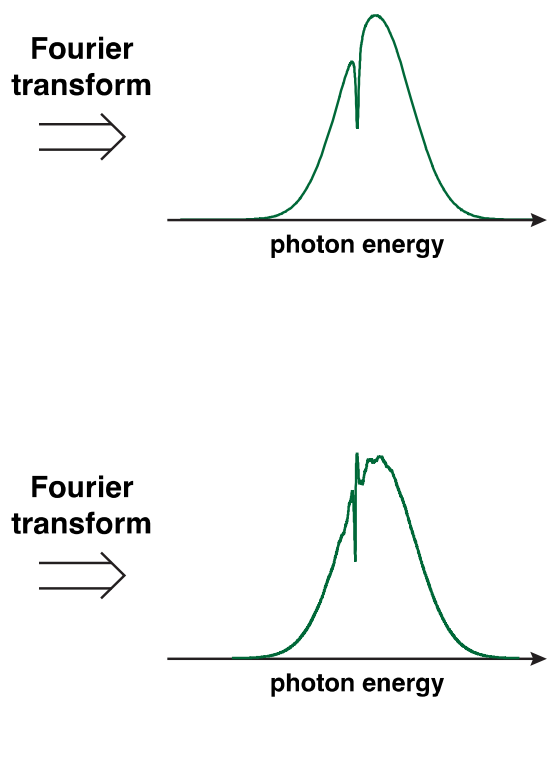

Absorption

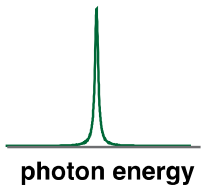

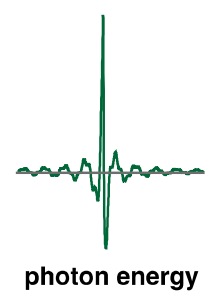

Figure 1: Simplified diagram of transient absorption. (a) XUV-only absorption measurement. The XUV pulse induces a polarization in the sample (left). The transmitted XUV spectrum (middle) shows a clear absorption feature and the calculated absorption (right) displays a single peak. (b) The NIR pulse can perturb the polarization at some delay (left), altering the transmitted spectrum (middle) and creating sidebands or altered absorption lineshapes in the expected absorption (right).

The temporal resolution of a transient absorption experiment depends on the duration of the laser pulses used in the experiment while the resolution in the frequency domain depends only on the energy resolution of the spectrometer, as described by Pollard and Mathies [5]. This is because the final absorption measurement, which is the signal at the detector integrated over all times, is not time-dependent. Therefore, attosecond transient absorption measurements can be performed with both excellent temporal resolution and good spectral resolution.

In conventional transient absorption spectroscopy, a weak broadband pulse is used to measure the absorption spectrum of a sample. A pump pulse initiates dynamics, and changes in absorption features are observed when the broadband pulse follows the pump pulse. These methods have been successfully extended to XUV photon energies. In the femtosecond experiments of Loh et al. [6], a femtosecond near-infrared (NIR) laser pulse is used to induce strong-field ionization in a sample. Afterwards, the absorption of the XUV pulse is measured, and changes in the absorption are observed as the sample becomes ionized. Analogous experiments using attosecond pulses instead of an XUV pulse train were initially proposed by Pfeifer et al. [7]. Later experiments using isolated attosecond pulses by Goulielmakis et al. [8] detected quantum beating in a wavepacket created in $\mathrm{Kr}^{+}$after strong-field ionization.

Attosecond transient absorption experiments can thus be divided into three distinct categories based on the relative time delay between the NIR and attosecond pulse: relative time delays when the NIR pulse precedes the attosecond pulse, when the NIR and attosecond pulses are overlapped in time, and when the NIR pulse follows the attosecond pulse. In conventional transient absorption experiments, the range of time 
delays when the NIR pulse follows the XUV pulse might be deemed uninteresting, as no dynamics can occur before the pump pulse has interacted with the system. However, important recent results in attosecond science have capitalized on transient absorption experiments in which the NIR pulse overlaps with or follows after the attosecond pulse. This Frontiers article focuses on the rich variety of dynamics that can be observed and characterized in these two regions of relative NIR/attosecond pulse time delay.

\section{Experimental techniques of attosecond transient absorption}

A generalized experimental apparatus for attosecond transient absorption is shown in Figure 2. Similar apparatuses used by other groups are described in detail by Chini et al. [9] and Ott et al. [10]. A NIR pulse (duration of approximately $7 \mathrm{fs}$ ) is first split by a beam splitter in an interferometer. One arm of the interferometer generates the attosecond pulse, while the other arm acts as the NIR probe pulse. The XUV pulse generating arm may pass through optics to implement an attosecond optical gating technique, such as Double Optical Gating [11], which manipulates the laser field in order to produce a single attosecond pulse. The pulse then enters the vacuum system and is focused into a gas cell, in which the high harmonic generation process occurs. After this gas cell, an XUV attosecond pulse is produced and propagates collinearly with the residual femtosecond laser field. This residual light is subsequently blocked by a metal foil, which also acts as a filter for the attosecond pulse spectral bandwidth. The attosecond pulse is then focused by a gold-coated grazing incidence toroidal mirror.

The NIR probe arm propagates outside the vacuum system. A relative delay can be introduced in this arm, and fused silica can be added for optimal pulse compression. In the apparatus shown here, the NIR pulse is first focused by a spherical focusing mirror and then is recombined with the XUV pulse by a mirror with a small hole drilled in the center. The XUV pulse passes through the hole in the center of the mirror, while the NIR pulse reflects off the mirror, resulting in an annular beam.

The NIR and XUV pulses are then overlapped spatially in a gas cell or jet where they interact with the sample. After passing through the sample, the NIR pulse is blocked by a metal foil. The XUV pulse is dispersed by a grating and its spectrum is detected,

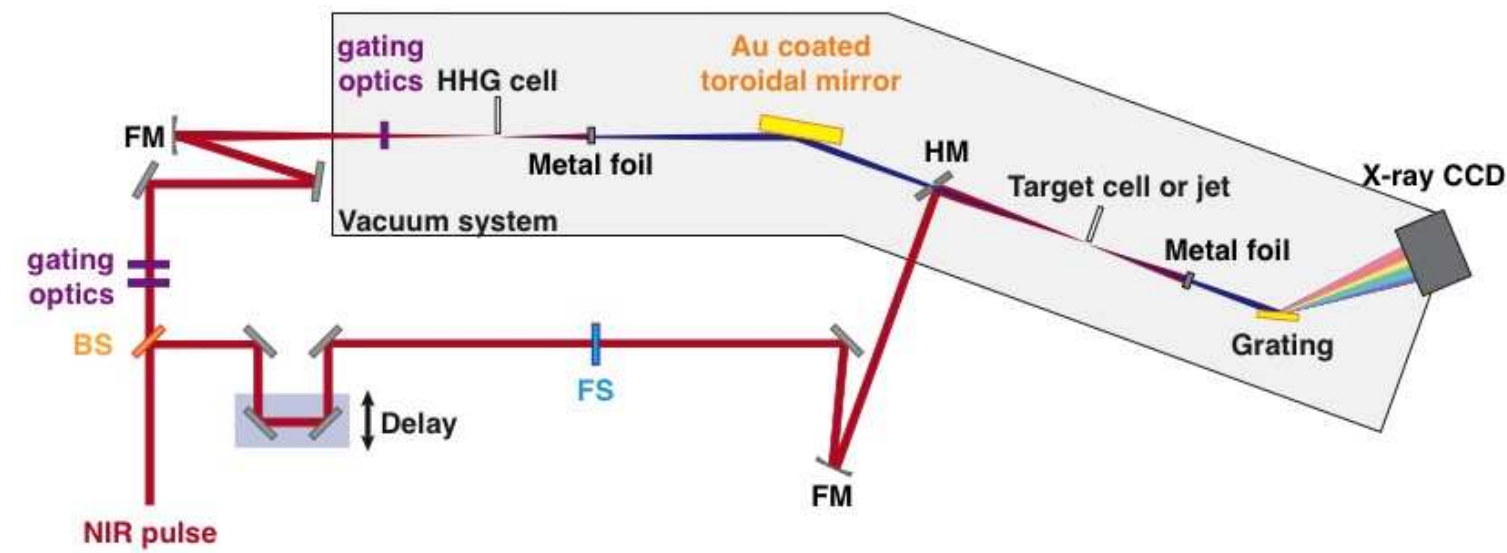

Figure 2: Typical attosecond transient absorption experimental setup. BS: beam splitter, FM: focusing mirror, HHG: high harmonic generation, FS: fused silica, HM: hole mirror. The outlined grey area represents the extent of the vacuum chambers. Details are outlined in the text. 
typically by an X-ray CCD camera.

A variation on this apparatus that has been used in some of the experiments discussed later in this article (e.g. Refs $[12,13])$ instead incorporates a compact interferometer that introduces the relative delay between the XUV and NIR pulses. Then, the two arms travel collinearly into the vacuum system. Both types of apparatus are versatile, as each arm of the interferometer can be manipulated separately.

\section{Induced polarization}

The induced polarization is a macroscopic sum of the individual oscillating dipoles of the atoms or molecules in the sample. The polarization is induced by the initial excitation, follows changes in the electric field near-instantaneously, and dephases over some timescale. This article will consider only experiments with gas phase targets. Transient absorption spectroscopy has also been performed in solid samples such as silicon $[4,14]$. However, dephasing of the polarization induced by the XUV pulse occurs much more rapidly in a solid than in a gas sample. For example, in silicon, the dephasing time of the XUV-induced polarization was estimated to be 100 as [14], while in a gas the dephasing time can be much longer than the timescales of typical attosecond transient absorption experiments. Due to this disparity in dephasing timescales, perturbation of the induced polarization by a few-femtosecond NIR pulse that follows the XUV pulse initially appears to be an effective measurement technique in the gas phase but not in solids. With shorter pulses, however, this counterintuitive regime of time delays could also be explored in solids.

The polarization induced in a sample is related to the electric field of the broadband pulse used for excitation. Applying the slowly varying envelope approximation (e.g. assuming that the spatial envelope of the pulse is large relative to the wavelength) gives the following relation between the field and the polarization:

$$
\frac{\partial \widetilde{\varepsilon}(\omega, z)}{\partial z}=i \frac{2 \pi \omega}{n c} \tilde{P}(\omega, z) e^{-i k t+i \omega t}
$$

where $\widetilde{\varepsilon}(\omega, z)$ is the field of the pulse, $\widetilde{P}(\omega, z)$ is the induced polarization, $n$ is the index of refraction of the material, and $k$ is the wavevector of the pulse, which propagates along the $z$ direction. If the polarization is uniform in space (namely, along the $z$ axis) the electric field is proportional to the polarization as

$$
\widetilde{\varepsilon}(\omega) \propto i \omega \widetilde{P}(\omega)
$$

In the treatment of Pollard and Mathies [5] the response of the system is separated into linear and nonlinear components. The linear component is the response of the system to the broadband pulse (the absorption of that pulse) and the nonlinear component reflects the effects of the pump pulse on the system. Then, the absorption cross-section is proportional to the polarization as:

$$
\sigma(\omega) \propto \omega \operatorname{Im}\left[\frac{\widetilde{P}^{(3)}(\omega)}{\widetilde{\varepsilon}(\omega)}\right]
$$

where $\tilde{P}^{(3)}(\omega)$ is the third-order component of the polarization. This is the lowest order that incorporates the effect of the pump pulse on the system, although higher order terms may also be considered. 
However, the response of the system may not necessarily be separable into components of different orders. A more general method [15] to calculate the transient absorption spectrum involves first calculating the single atom response function as:

$$
\tilde{S}(\omega)=2 \operatorname{Im}\left[\tilde{\mu}(\omega) \widetilde{\varepsilon}^{*}(\omega)\right]
$$

where $\tilde{\mu}(\omega)$ is the Fourier transform of the time-dependent dipole moment and $\widetilde{\varepsilon}^{*}(\omega)$ is the Fourier transform of the electric field of the XUV. The macroscopic polarization, or the polarization of the entire sample, can be approximated as:

$$
\tilde{P}(\omega)=2 \rho \tilde{\mu}(\omega)
$$

where $\rho$ is the number density of the sample. Thus, the dipole moment can be considered as a proxy for the polarization, and the single atom response function approximates the measured transient absorption spectrum. The key differences between the single-atom response and the effects due to propagation through a sample composed of many atoms will be discussed in section 4.3. If the polarization of the medium is simply calculated by summing the single-atom response functions, the cross-section is then given by

$$
\sigma(\omega) \propto \omega \operatorname{Im}\left[\frac{\tilde{\mu}(\omega)}{\widetilde{\varepsilon}(\omega)}\right]
$$

Considering the relationship between the Fourier transform of the time-dependent dipole moment and polarization in Equation [5], this expression for the absorption cross-section agrees with the expression given in Equation [3] but is more generally applicable. This mathematical treatment can then be used to model the effect of the NIR pulse on the system, and it will be applied to specific cases in the following sections.

\section{Regimes of attosecond transient absorption spectroscopy}

\subsection{Time overlap of the NIR and XUV pulses}

Dynamics observed when the NIR and XUV pulses are overlapped in time can be broadly separated into two categories: a coupling between states that is induced by the NIR pulse and an interference between multiple pathways leading to the same final state. Processes in the former category include Autler-Townes splitting and the observation of light-induced states (LISs), while interference between two pathways can manifest as subcycle interference fringes.

The helium atom is a much-studied system, due to the ease of theoretical calculations and the convenience of use in experiments. Transient absorption experiments were performed on helium in conjunction with time-dependent Schrödinger equation (TDSE) calculations by the Schafer group [16]. In both the experimental measurements and the theoretical work, new features that do not correspond to an allowed atomic absorption are observed when the NIR and XUV pulses are overlapped. These features are assigned as light-induced states (LISs), which become visible in the absorption spectrum as a result of population transfer from the ground state $\left(1 s^{2}\right)$ to dark states $(1 s \mathrm{n} s$ or $1 s \mathrm{n} d)$ via a resonant process requiring one photon of the XUV and one photon of the NIR. The LIS acts as the intermediate state in this process, which can be thought of as an analog to Raman spectroscopy. This mechanism was confirmed by calculations in which the $s$ and $d$ states were removed from the calculation and, as a result, no LISs are observed. 
(a)
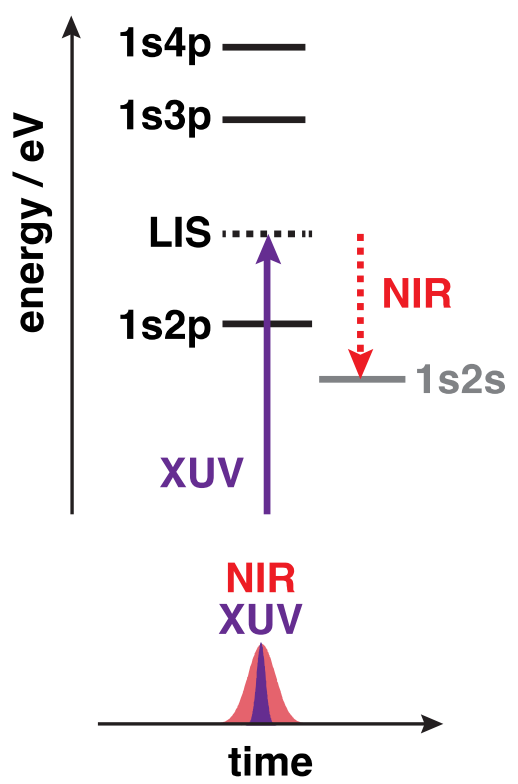

(b)

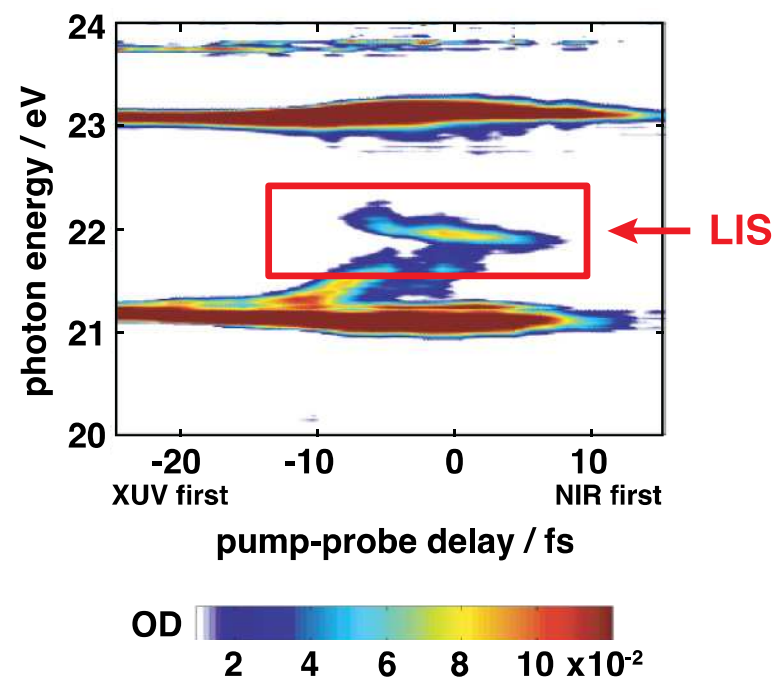

Figure 3: Light-induced states (LISs). (a) Energy level diagram showing a LIS in helium. Purple solid arrow: XUV pulse, red dotted arrow: NIR pulse. Levels that can be accessed by allowed transitions from the ground state are shown as black horizontal lines, dark states are grey, the LIS is dotted. The energy level positions are aligned with the experimental data. (b) Attosecond transient absorption data showing a LIS (marked by red box). Negative time delays: XUV pulse precedes NIR. Adapted from [16].

Figure 3(a) shows the mechanism that creates a LIS that couples the ground state to the dark $1 s 2 s$ state, and Figure 3(b) shows the experimental observation of that LIS. Observation of LISs in a transient absorption spectrum allows the dark states to be located in energy and identifies which coupling pathways are dominant in the system under consideration. LISs could also be used to transfer population to dark states that are not accessible by one-photon transitions from the ground state.

Another effect that also results from NIR coupling to a dark state is AutlerTownes splitting [17], which occurs when the NIR photon energy is close to resonant with the transition to a dark state. A single absorption feature is split into a doublet as a result of strong-field coupling between the initially excited state and a dark state. This splitting is analogous to the phenomenon of electromagnetically induced transparency (EIT) in x-ray studies. The splitting of an absorption feature into a doublet results in increased transmission of the XUV light at the original position of the resonance. EIT has been studied theoretically [18-20] and experimentally observed using femtosecond XUV pulses [21,22]. EIT as well as more complicated splitting of absorption features in XUV transient absorption was noted in calculations by Gaarde et al. [15]. The observation of this splitting instead of LISs could distinguish which possible coupling pathways are most important using a certain probe pulse. EIT could also be used to manipulate the spectrum of an XUV pulse, by creating holes in the spectrum at resonance positions. 
Although the coupling mechanisms by which LISs and Autler-Townes splitting are observed are similar, the most obvious difference between these two phenomena is whether a change in absorption is observed at the position of a bright state (AutlerTownes) or at a photon energy that does not correspond to an allowed one-photon transition from the ground state (LIS). Which effect is observed depends on the spacings between the energy levels in the target system relative to the photon energy of the NIR pulse. If the NIR pulse is close to resonant with the transition between an initially excited state and a dark state, Autler-Townes splitting is observed. However, as the position of the dark state moves away from resonance with the NIR photon energy, a LIS is observed instead. This is shown in Figure 4 for two cases: Figure 4(a) and (c) show Autler-Townes splitting, occurring when the NIR pulse is resonant with a transition

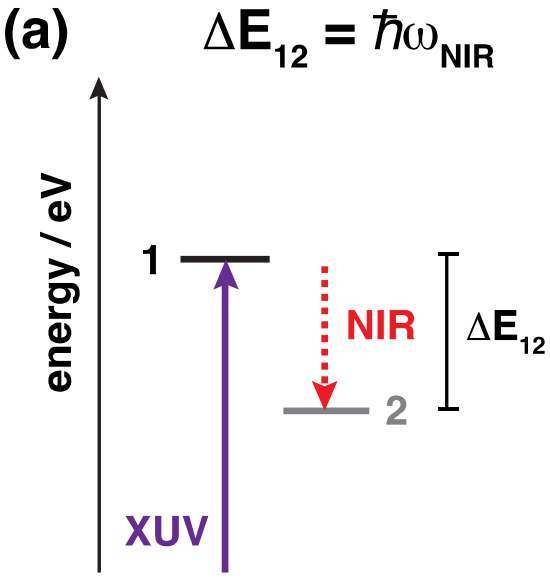

(c)

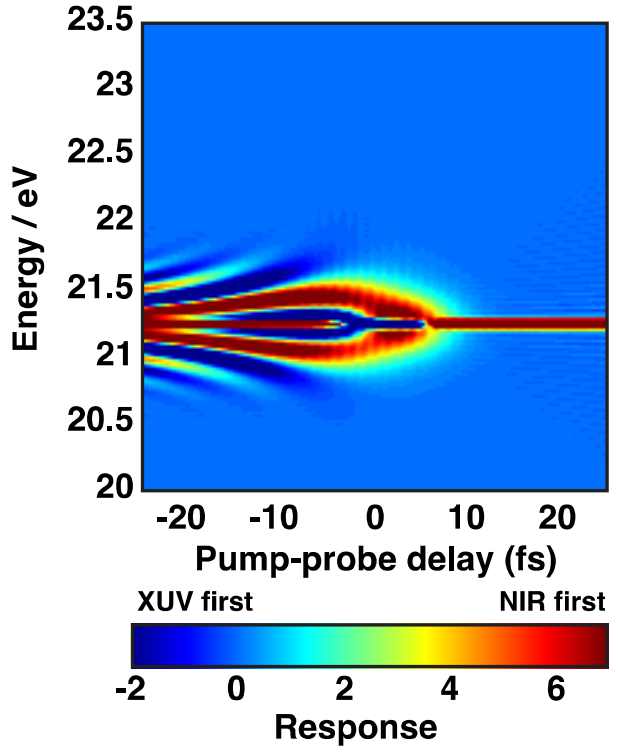

(b)

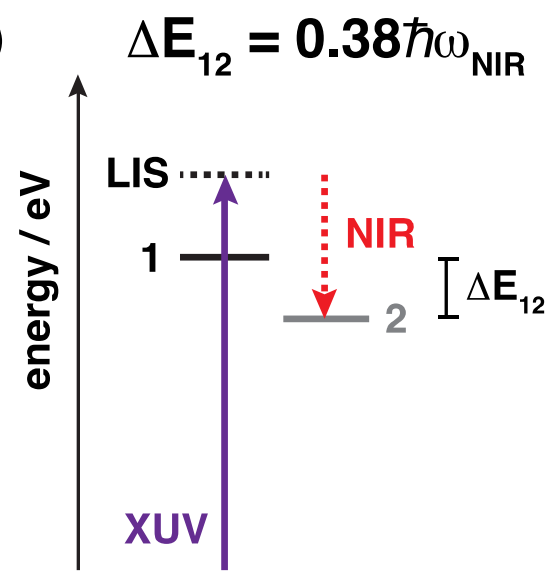

(d)

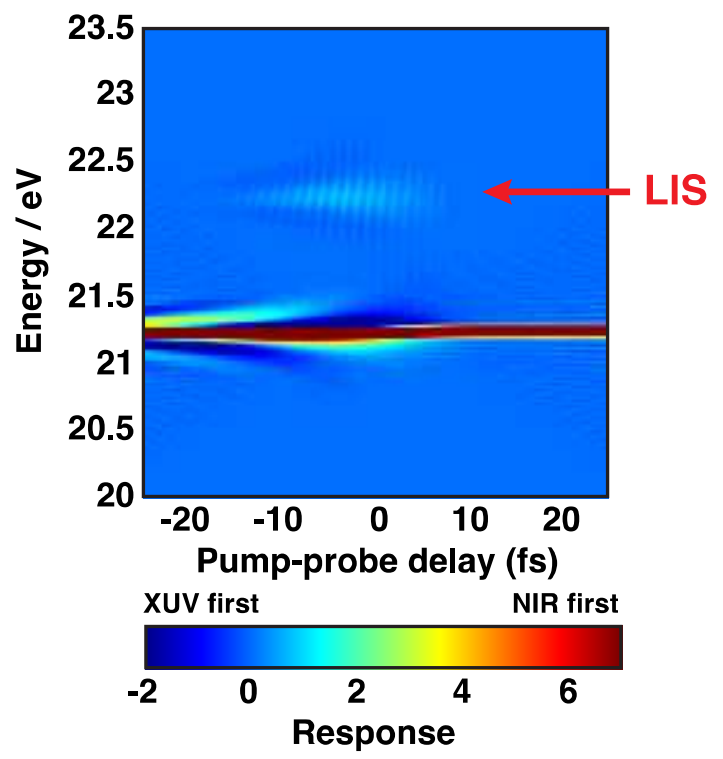

Figure 4: Regimes of Autler-Townes splitting and LIS. (a) The NIR pulse $(800 \mathrm{~nm}$ central wavelength, red dotted arrow) is resonant with a transition between state 1 , which is excited by the XUV (purple solid arrow) and state 2, which is a dark state. (b) The NIR pulse is no longer resonant with this transition (the splitting between states 1 and 2 is $0.38 \hbar \omega_{N I R}$ ). (c) Calculated transient absorption result for case shown in (a). Negative time delays: XUV pulse precedes NIR. (d) Calculated transient absorption for case shown in (b). The position of the LIS is marked with an arrow. Calculations used with permission of Adrian Pfeiffer. 
between a bright state and a dark state. Figure 4(b) and (d) show a LIS becoming visible instead when the NIR is far from resonant with this transition. This transition from Autler-Townes splitting to the appearance of the LIS could be probed by scanning the wavelength of the probe pulse, which would also identify the dominant coupling pathways to dark states.

The LISs were theoretically predicted to be sensitive to the NIR intensity [16], and experiments characterized the intensity dependence of the LIS in helium [23]. The discrepancy between the shifts in the LIS position predicted by theoretical work and the shifts observed in experiments was attributed to the difficulty of accurately determining the NIR intensity in the experimental measurements.

The Stark shift induced by the NIR pulse can also induce a change in the absorption lineshape, which changes because the NIR field causes a phase shift in the induced dipole moment. This effect was treated theoretically by the groups of Schafer [24] and Santra [25] for long-lived states and by Chu and Lin [26] for autoionizing states. Ott et al. [10] first characterized this effect experimentally, showing that the Lorentzian lineshapes of absorption features in helium corresponding to longlived atomic states can be converted to Fano profiles, and vice versa. The phase shift depends on the NIR intensity and duration, and is therefore delay-dependent. An example of this NIR-induced lineshape change in the absorption of Rydberg states of neon is shown in Figure 5. The top panel of Figure 5 shows the XUV-only absorption, in which the features appear as weak, narrow peaks, and the bottom panel shows the absorption spectrum when the XUV and NIR pulses are temporally overlapped and the absorption lineshapes have become Fano-like. This laser-induced phase is a clear example of how the NIR pulse can be used to precisely manipulate the absorption spectrum. The observed Fano lineshape parameter of the absorption line also characterizes the magnitude of the phase shift induced by the NIR pulse.

Other features observed in attosecond transient absorption measurements when the NIR and XUV pulses are overlapped in time are due to interference between two

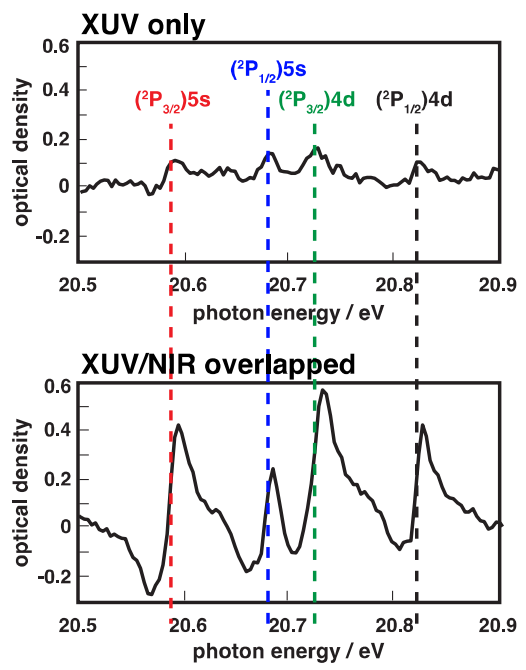

Figure 5: Lorentz to Fano lineshape change in neon. Top panel: XUV-only absorption (Rydberg level positions marked with dotted lines). Bottom panel: Absorption with XUV and NIR pulses overlapped in time. 
pathways that both result in the same final state. Subcycle interference fringes were predicted theoretically by the Schafer group [16,27] and observed in transient absorption measurements performed by Chini et al. [9] and X. Wang et al. [28]. These fringes have a period of approximately $1.3 \mathrm{fs}$, which is half the NIR laser cycle ( $2.6 \mathrm{fs})$. The fringes arise from interference between a direct pathway and an indirect pathway. The direct pathway is merely absorption of one XUV photon, transferring population from the ground state to an excited electronic state. However, population can also be transferred to the same excited state by an indirect pathway: a lower-lying state is excited by the XUV and then two NIR photons couple the two states. A schematic of these interference pathways is shown in Figure 6(a), and the calculated single-atom response function for helium, which clearly displays subcycle interference fringes, is shown in Figure 6(b). These interferences are observed in both LISs, which are only visible during the overlap of the XUV and NIR pulses, and in long-lived electronic states, where the subcycle features remain visible when the NIR pulse follows the XUV pulse.

The origin of these features was confirmed theoretically by removing states from the calculation and noting the resulting disappearance of the subcycle features. An analogous experiment was performed by X. Wang et al. [28], who compared the transient absorption spectrum of neon recorded using a broadband XUV pulse, which can excite a wide range of Rydberg states between $16 \mathrm{eV}$ and the ionization potential at $21.56 \mathrm{eV}$, to a measurement using a narrowband XUV pulse, which excludes excitations to all states except the $2 s^{2} 2 p^{5} 3 s$ states. Subcycle interference fringes were clearly observed with the broadband excitation pulse but disappeared in the narrowband transient absorption

(a)

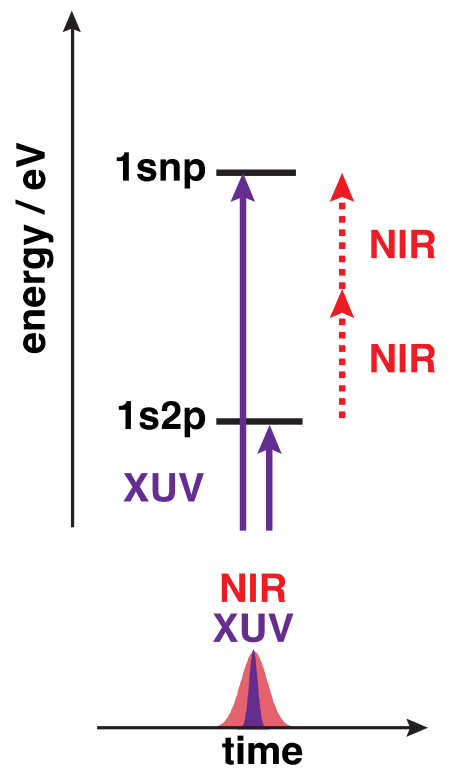

(b)

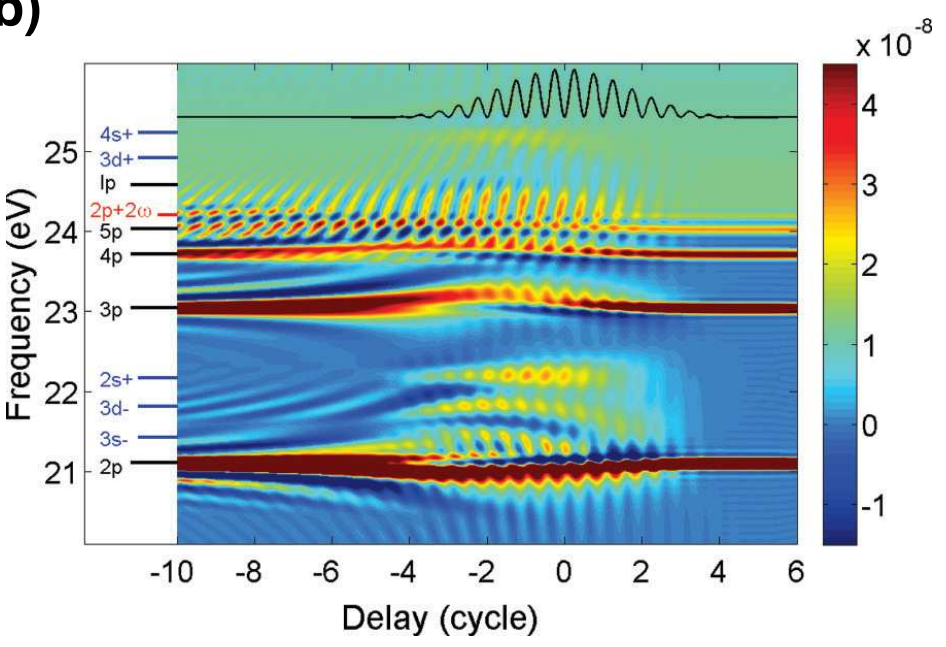

Figure 6: Subcycle interference fringes in transient absorption. (a) Energy level diagram of helium, showing two pathways that interfere to produce the fringes. Purple solid arrows: XUV photon energies, red dotted arrows: NIR photon energy. (b) Calculated single atom response of helium, showing subcycle fringes. Negative time delays: XUV pulse precedes NIR. Figure from Ref. [27]. Reprinted figure with permission from S. Chen et al., Phys. Rev. A, 87, 033408 (2013). Copyright 2013 by the American Physical Society. 
measurement. These subcycle interference fringes indicate that the population in the system is being manipulated on an attosecond timescale, as the NIR pulse transfers population between the initially excited levels.

The population transfer induced by the NIR pulse can be enhanced if there is an atomic or molecular level that can act as a near-resonant intermediate state. Chen $e t$ al. [27] have shown theoretically that information about the position of this intermediate state can be extracted from the subcycle interference fringes. The phase of the interference fringes depends on the magnitude of the NIR photon energy relative to the difference in energy between the initial state and the intermediate state in this process. Experimentally, using NIR pulses with varying central wavelength could identify the intermediate state in the coupling process.

Subcycle features can also be observed in LISs, and the appearance of the fringes identifies which dark state produces each LIS. The sign of the slope of the fringes with respect to delay indicates whether the LIS is higher or lower in energy than the final dark state [27].

Theoretical work performed by Pfeiffer et al. [29] and Wu et al. [30] has shown that the presence of subcycle features in calculated transient absorption spectra depends on the inclusion of counter-rotating terms in the expression for the dipole moment. In theoretical models, the rotating wave approximation (RWA) is often used. Counterrotating terms that oscillate rapidly, or are small near resonance, may be excluded to simplify the mathematics. This means that terms with frequency $\omega=\omega_{0}+\omega_{I R}$, where $\omega_{0}$ is the transition frequency and $\omega_{I R}$ is the laser frequency, are removed, while terms

(a)

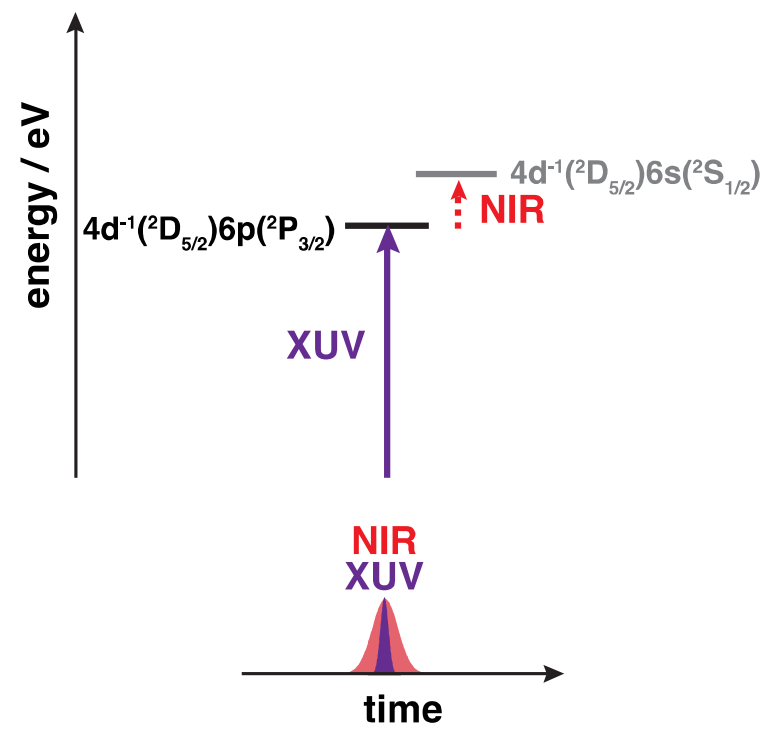

(b)

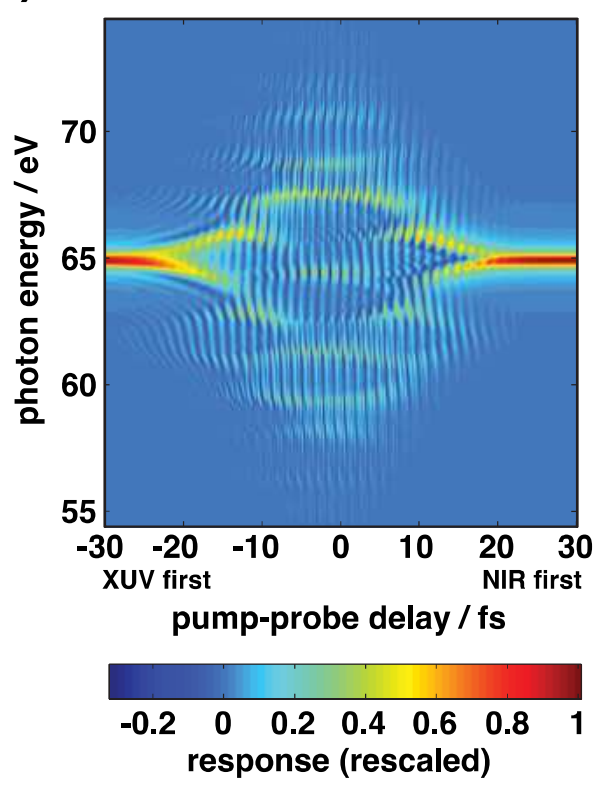

Figure 7: Autler-Townes splitting in xenon. (a) Energy level diagram for xenon. Purple solid arrows: XUV photon energy, red dotted arrows: NIR pulse coupling to dark state. States accessible by one-photon excitation from the ground state are shown as black horizontal lines, and dark states are grey lines. (b) Full calculation of polarization response. Subcycle features are clearly visible. Negative delays: XUV pulse precedes NIR. Adapted from [29]. 
with frequency $\omega=\omega_{0}-\omega_{I R}$ are kept. However, when the ratio of the Rabi frequency $\Omega_{R}$ to the laser frequency is large, the RWA no longer accurately reproduces all of the physical effects. The Rabi frequency is defined as $\Omega_{R}=d_{0} E_{I R}$, where $d_{0}$ is the transition dipole moment and $E_{I R}$ is the amplitude of the electric field, so the RWA breaks down if the NIR intensity is high or the coupling between states is strong.

The effect of the RWA was shown in calculations of Autler-Townes splitting in helium [30] and xenon [29]. The magnitude of the splitting depends on the intensity of the NIR field, which is therefore dependent on the NIR-XUV delay. Calculations using the RWA accurately reproduced the overall delay dependence of the Autler-Townes splitting and other structures that were observed in experimental measurements. However, the subcycle features did not appear in these calculations. When instead a full time-dependent Schrödinger equation (TDSE) calculation was performed, the subcycle features appeared clearly, as shown in Figure 7. Another effect that could not be reproduced in calculations using the RWA is the splitting of the absorption features into multiple branches instead of merely into a doublet. This multiplet splitting has been experimentally observed in femtosecond transient absorption measurements of core excited xenon, using $100 \mathrm{fs}$ NIR pulses and intensities on the order of $10^{14} \mathrm{~W} / \mathrm{cm}^{2}$ [31]. Multiplet splitting was also proposed as a possible explanation for new features observed in LISs in helium as the NIR intensity was increased, although the spectral resolution was not sufficient to resolve the possible splittings [23]. Using the RWA in theoretical calculations does not fully predict the phenomena that will be observed in an experiment, and full TDSE calculations are necessary to correctly predict or replicate experimental attosecond transient absorption results.

\subsection{The NIR pulse follows the XUV pulse}

Experiments in which the NIR pulse follows the attosecond pulse at large time delays (ranging from only a few fs to tens or hundreds of fs) provide an unprecedented wealth of information about electronic dynamics. Inducing a polarization with the XUV pulse and then perturbing the system with a NIR pulse that follows at delays of a few femtoseconds or longer has been applied to measure decay timescales of autoionizing states and to characterize quantum beating between electronic states.

A recent, very effective application of attosecond transient absorption spectroscopy is directly measuring the lifetimes of short-lived atomic states in the time domain. Autoionizing states were excited in argon atoms by $\mathrm{H}$. Wang et al. [32] and in xenon atoms by Bernhardt et al. [12]. The absorption features corresponding to shortlived autoionizing states embedded in the continuum appear in the spectrum as Fano resonances. The lifetimes of these states were previously estimated from high-resolution synchrotron measurements [33] to be on the order of tens of fs. Therefore, these states are an excellent test case for comparison of the lifetime determined from the linewidth with the lifetime that is directly measured. The decay of the autoionizing state is monitored by interacting a time-delayed NIR pulse with the target. The initially strong absorption feature is depleted when the NIR and XUV pulses are overlapped, because the NIR pulse couples population out of the initially excited state either to other atomic states or to the ionization continuum. This produces a broadened and weakened absorption feature. The absorption feature then slowly recovers. A schematic of the coupling 
(a)
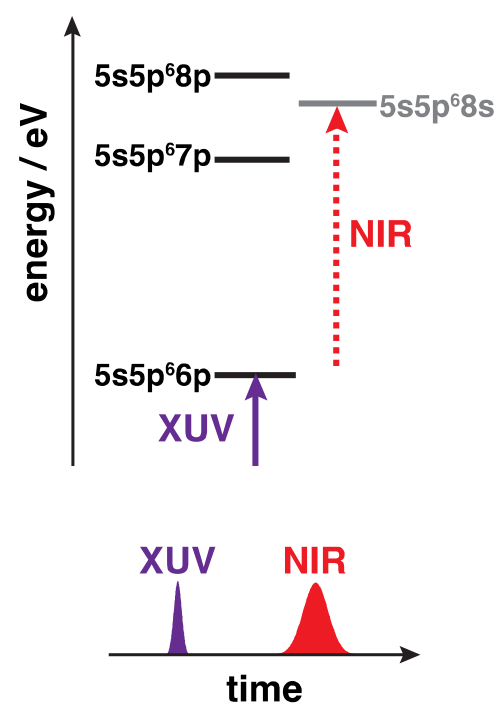

(b)

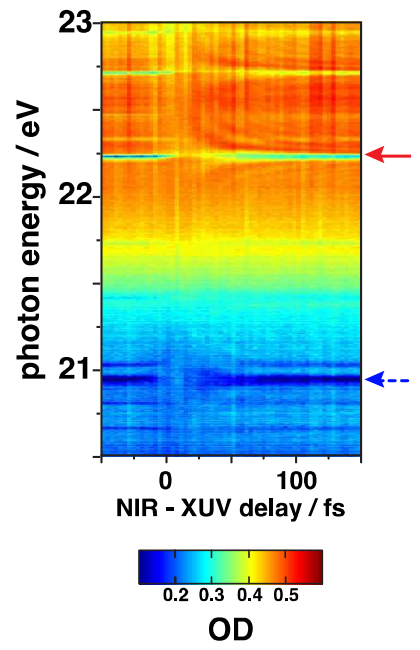

(c)

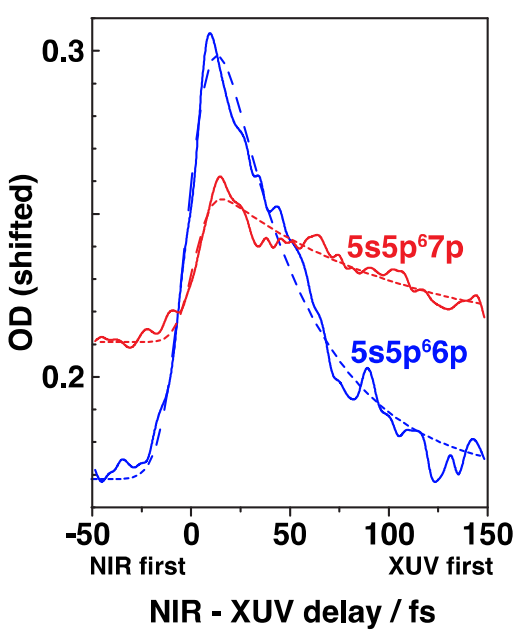

Figure 8: Measurement of lifetime of autoionizing state by coupling to dark state. (a) Energy level diagram for xenon. Purple solid arrow: XUV photon energy to excite the $5 s 5 p^{6} 6 p$ state, red dotted arrow: NIR pulse coupling to dark state (grey). (b) Experimental measurement of lifetimes of autoionizing states in xenon. Color scale: OD (optical density). Negative time delays: NIR pulse precedes XUV. Arrows mark lineout positions (blue dashed: $5 s 5 p^{6} 6 p$, red solid: $5 s 5 p^{6} 7 p$ ). (c) Lineouts at center of autoionizing state absorption features. Blue solid line: $5 s 5 p^{6} 6 p$ data, blue dashed line: $5 s 5 p^{6} 6 p$ fit, red solid line: $5 s 5 p^{6} 7 p$ data, red dashed line: $5 s 5 p^{6} 7 p$ fit. Adapted from [12].

process is shown in Figure 8(a), and experimental measurements of the lifetimes of $5 s 5 p^{6} \mathrm{n} p$ states in xenon are shown in Figure 8(b).

The timescale of the recovery of the absorption feature is actually twice the lifetime of autoionizing state. This factor of two arises because the absorption signal is proportional to the polarization rather than the population. This can be derived by calculating the absorption spectrum using Equation [4]. A simple model system will be considered where there are only two states, the ground state (denoted as state 0 ) and an excited state (denoted as state 1 ) that is short-lived, with a lifetime $1 / \Gamma$. The population in the excited state after an initial excitation is thus

$$
\left|\psi_{1}(t)\right|^{2}=e^{-\Gamma t}\left|\psi_{1}(0)\right|^{2}
$$

which decays exponentially with the state lifetime of $1 / \Gamma$. However, the quantity needed to evaluate the time-dependent dipole moment is the wavefunction. The time-dependent wavefunction of this state is

$$
\left|\psi_{1}(t)\right\rangle=e^{-i \omega_{1} t} e^{-\left(\frac{\Gamma}{2}\right) t}\left|\psi_{1}(0)\right\rangle
$$

where $\omega_{1}$ is the energy of the excited state. The factor of $\Gamma / 2$ in the exponential ensures that the population decay occurs on the correct timescale, as in Equation [7]. This critical factor will be the source of the factor of two difference in the population and polarization decay. The total wavefunction for the system, after excitation by the XUV only, is

$$
|\Psi(t)\rangle \approx C_{0}(0) e^{-i \omega_{0} t}\left|\psi_{0}\right\rangle+C_{1}(0) e^{-i \omega_{1} t-\left(\frac{\Gamma}{2}\right) t}\left|\psi_{1}\right\rangle
$$

where $\omega_{0}$ is the energy of the ground state. $C_{0}$ and $C_{1}$ are coefficients with some time dependence. If only a small fraction of the initial ground state population is excited, 
$C_{0} \approx 1$. The time-dependent dipole moment is evaluated, keeping only terms that are large near the resonance center frequency, and modeling the effect of the NIR pulse as a sudden annihilation of the dipole moment at a delay time $\tau$. This time-dependent dipole moment is then

$$
\mu(t)=\left\{\begin{array}{lr}
C_{1}(0)\left\langle\psi_{0}|\hat{\mu}| \psi_{1}\right\rangle e^{-i\left(\omega_{1}-\omega_{0}\right) t-\left(\frac{\Gamma}{2}\right) t}, & 0<t<\tau \\
0, & \tau \leq t
\end{array}\right.
$$

The quantity of interest is the response function versus frequency, so the time-dependent dipole moment must be Fourier transformed. The absorption on the resonance center, or when $\omega=\omega_{1}-\omega_{0}$, is then given by:

$$
\tilde{S}\left(\omega_{1}-\omega_{0}\right) \propto 1-\mathrm{e}^{-\left(\frac{\Gamma}{2}\right) t}
$$

The absorption thus decays exponentially with a time constant of $2 / \Gamma$, or twice the state lifetime. This factor of two difference, which only appears in measurements of decay timescales, is critical to recognize in order to accurately extract a lifetime from transient absorption measurements. Fitting the temporal profile of the depletion and recovery to this functional form allows the lifetime of the autoionizing state to be determined by a direct measurement in the time domain.

The lifetimes measured by attosecond transient absorption spectroscopy agree with lifetimes calculated from linewidths, using the uncertainty principle, in synchrotron measurements. However, absorption features of short-lived states in molecules can overlap in energy or undergo additional broadening processes, so this technique could be applied to directly measure these short lifetimes that may not be accurately extracted from static absorption spectra.

When considering the temporal evolution of the absorption at a small detuning from the resonance center, more complicated behavior is observed. Incorporating a detuning $\Delta \omega=\omega-\left(\omega_{1}-\omega_{0}\right)$ into the expression for the dipole moment and calculating the single-atom response as above results in a function of the form

$$
\tilde{\mathrm{S}}(\omega) \propto \cos (\Delta \omega t) e^{-\frac{\Gamma}{2} t}
$$

For photon energies at a small detuning from the resonance center, there is an additional cosine term, which creates oscillations in energy for a given time delay. This is a manifestation of perturbed free induction decay, which occurs when the ringing of a dipole is suddenly cut off $[34,35]$. In a transient absorption measurement, this effect produces hyperbolic sidebands with spacing inversely proportional to the NIR-XUV time delay. These sidebands are observed in transient absorption measurements such as those shown in Figure 8 or Figure 9.

Transient absorption measurements when the NIR pulse couples population to the continuum versus to another discrete state are compared theoretically in Li et al. [36]. The calculated transient absorption spectra look similar for low NIR intensities (approximately $10^{12} \mathrm{~W} / \mathrm{cm}^{2}$ ). As the NIR intensity increases, the absorption lineshape when the NIR and XUV pulses are overlapped in time varies dramatically depending on which coupling mechanism is occurring. If the NIR pulse is coupling to the continuum (e.g. ionizing the state), the absorption feature disappears as the NIR intensity increases because the population becomes completely ionized. However, if the NIR is coupling to a discrete state, splittings are observed in the absorption feature. Additionally, the time dependent behavior of the absorption at the resonance center also varies depending on which coupling case is under consideration. For instance, Rabi cycling is observed at 
high NIR intensities if the NIR couples the initially excited state to another discrete state, but not if the NIR pulse is only ionizing the state. NIR intensity dependent measurements can therefore determine the effect of the NIR pulse on the system.

The broadband attosecond pulse can also be used to excite superpositions of multiple electronic states, and the resulting quantum beats can be observed using attosecond transient absorption. This effect was predicted in calculations by Chini et $a l$. [37] showing that beating between pairs of $\mathrm{n} p$ states in helium could be observed in the transient absorption spectrum. Quantum beating was then experimentally observed in long-lived atomic states by exciting a wavepacket composed of Rydberg states of neon [13]. Multiple quantum beats were observed, with periods ranging from 10 fs to 40 fs. The most prominent quantum beat observed was between the $2 s^{2} 2 p^{5}\left({ }^{2} \mathrm{P}_{3 / 2}\right) 3 d$ and $2 s^{2} 2 p^{5}\left({ }^{2} \mathrm{P}_{1 / 2}\right) 3 d$ levels, which are split in energy by $0.1 \mathrm{eV}$. The measured oscillation period was $40 \mathrm{fs}$, which agrees with the expected oscillation period due to this energy splitting. Figure 9(a) is an energy level diagram of neon, focusing on the $3 d$ states, and experimental measurements of quantum beating in the spin-orbit split $3 d$ levels of neon are shown in Figure 9(b). In contrast to the measurement of decay lifetimes described above, in which the decay of the polarization and of the population differ by a factor of two, the beating period observed via probing the polarization agrees with the beating period that would be observed by detecting the population. This is because the beating period depends only on the energy difference between the two states, which is the same whether polarization or population is considered.

In this measurement, several experimental artifacts are observed due to saturation

(a)
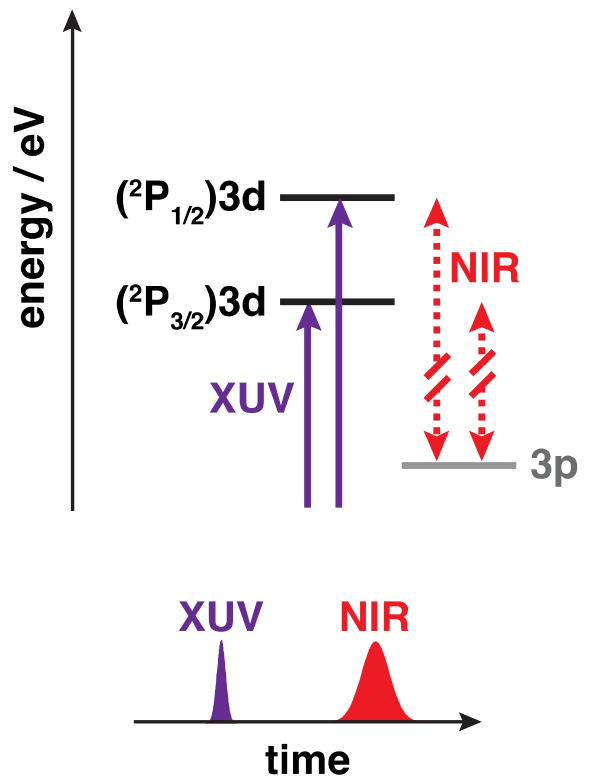

(b)
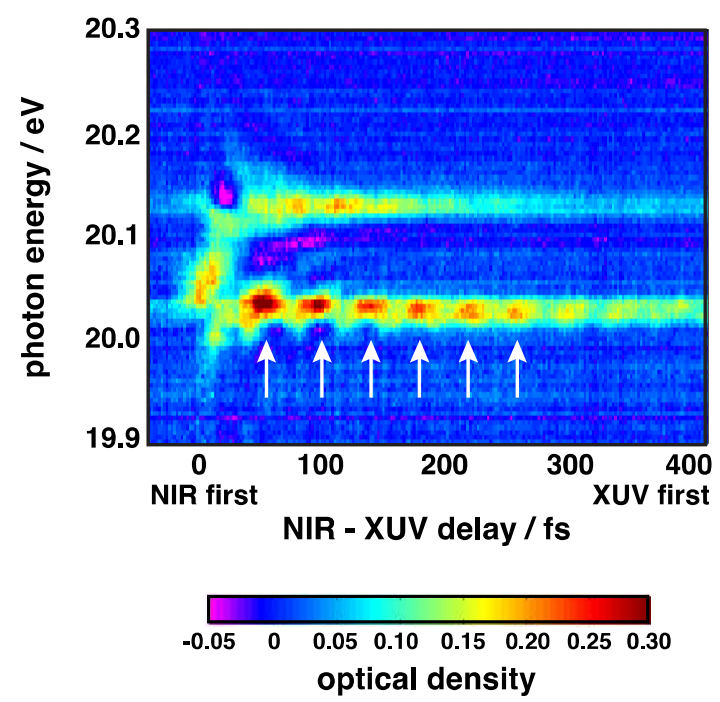
of the absorption feature and the limited spectral resolution of the detector. The absorption appears to decay on a timescale of hundreds of femtoseconds, although the lifetimes of the atomic states are on the order of nanoseconds, and each absorption peak appears stronger when the NIR pulse follows the XUV pulse than in the XUV-only absorption spectrum. These effects are both observed because the NIR pulse artificially shortens the lifetime of the state when it arrives at a given time delay. This shorter lifetime results in a broader absorption feature, and the absorption feature is saturated so the broadening also produces an apparent increase in absorption as the wings of the feature spread to neighboring pixels on the detector. The apparent decay in absorption strength over the timescale of the experiment is then just the decrease in the broadening of the feature as the delay of the NIR pulse increases.

The quantum beating is observed because the NIR pulse couples the initially excited states to neighboring dark states, and the effect of the NIR pulse is to Rabi-cycle population between the states. This mechanism is shown in Figure 9(a). The NIR pulse couples the $3 d$ states to $3 p$ states located about $1.5 \mathrm{eV}$ lower in energy, which closely matches the NIR pulse central photon energy. The strength of the beating visible in each feature depends on the strength of the couplings between the initially excited states and the dark states, thus explaining why the modulation depth of the beating could be strong in one state and weak in the other. The appearance of the beating was also shown theoretically to depend on which dark states are accessible within the NIR pulse bandwidth, which explains variations from day to day as the NIR pulse spectrum can fluctuate.

The observation of quantum beating can also be described as a manifestation of interference between two excitation pathways in analogy to the subcycle interference features discussed in section 4.1. One pathway requires one XUV photon to access the $2 s^{2} 2 p^{5}\left({ }^{2} \mathrm{P}_{1 / 2}\right) 3 d$ state, one NIR photon to access a dark state (most likely a $3 p$ state), and a second NIR photon to couple from this $3 p$ state to the $2 s^{2} 2 p^{5}\left({ }^{2} \mathrm{P}_{3 / 2}\right) 3 d$ state. The other pathway is merely direct excitation of the $2 s^{2} 2 p^{5}\left({ }^{2} \mathrm{P}_{3 / 2}\right) 3 d$ state by one XUV photon. The energy difference between these pathways is exactly the energy difference between the two states that compose the initial wavepacket. Therefore, the spacing of the resulting interference fringes is the same as the quantum beating period, both corresponding to the energy difference between the two states. This interpretation of quantum beating is confirmed in the recent work of Ott et al. [38], who observed the beating of a wavepacket composed of doubly excited states of helium. Here, the beating period is $1.2 \mathrm{fs}$, which is approximately the same as the expected spacing of the subcycle interference fringes. The energy separation between the two states composing the wavepacket corresponds to the energy of two NIR photons, and thus the coupling pathways that allow the beating to be observed are indistinguishable from the general mechanism for observation of subcycle interference fringes.

Quantum beating in the $5 s 5 p^{6} 6 p$ autoionizing state of xenon has also been observed [36]. The measured oscillation period of approximately $30 \mathrm{fs}$ corresponds to an initially excited wavepacket composed of the $5 s 5 p^{6} 6 p$ state, located at $20.95 \mathrm{eV}$, and a neighboring two-electron excited state located $140 \mathrm{meV}$ lower in energy. By comparison to few-level theoretical models, the intermediate state involved in the NIR coupling process could be identified as the $5 s 5 p^{6} 8 s$ state. 

systems with dense absorption spectra. Exciting a wavepacket and then monitoring individual components can allow the extraction of the lifetimes of the individual states involved in the wavepacket, which would again display a factor of two difference between the decay timescale in the absorption measurement and the population lifetimes of the states. These lifetimes of electronically excited states are determined by electronelectron interactions. The strength of the visible beating in each feature could provide information about the coupling strengths between these states and nearby dark states, which opens up new capabilities to use lasers to not just passively observe an atom or molecule, but to directly manipulate its electronic states.

\subsection{Incorporating propagation effects}

In the majority of the calculations discussed above, the transient absorption spectrum is initially calculated using Equation [4], which applies to a single atom. However, the experimental measurements are performed with an ensemble of target atoms. The differences between the single atom picture and the macroscopic measurement have been explored theoretically, in order to calculate transient absorption spectra that are in agreement with experimental results, and theoretical work shows that the correct transient absorption spectrum cannot necessarily be calculated by merely summing the single atom response over many atoms.

Attosecond transient absorption experiments are typically performed using low sample densities and short propagation distances, which have been shown by Chen et al. to result in absorption spectra very similar to the single-atom response alone [24]. Santra et al. [39] have shown that Beer's Law can accurately describe the absorption spectrum after passing through the sample if the temporal structure of the XUV pulse does not get significantly distorted after propagation, or if each frequency component propagates independently.

There are two main mechanisms that can reshape the XUV pulse during propagation. First, Beer's Law breaks down if there are couplings between states, e.g. if the sample can absorb at a frequency $\omega_{1}$ and then emit at a frequency $\omega_{2}$ [39]. Second, with increased sample densities, the XUV pulse can be reshaped after propagation through the medium due to the dispersion induced by strong absorption resonances. This reshaping has been previously described for the general case of a broadband pulse propagating through a medium with a strong absorption [40]. It was observed to occur in XUV pulses by Strasser et al. [41], in whose experiments photoelectron yields from helium at varying target pressures were measured using an XUV pulse train as the pump and a NIR probe pulse. The time dependent behavior of the photoelectron yield was found to depend on the gas pressure. The temporal structure of the XUV pulse exhibited periodic variations in amplitude over longer times, and this structure could be accurately reproduced by simple calculations of the pulse propagation considering the dispersion of the medium.

Of course, reshaping the XUV pulse structure will also affect transient absorption measurements. The absorption lineshapes can change in a similar manner to the NIRinduced phase shift discussed in section 4.1, but in this case the origin of the change in lineshape is the structure of the reshaped XUV pulse itself. Theoretical work by Chen et 
al. [24] has shown a noticeable effect due to reshaping of the XUV pulse after only a 1 $\mathrm{mm}$ propagation distance through 3 Torr of helium, with more drastic effects if the gas pressure is increased by a factor of 5.

The effect of the NIR pulse can also vary with propagation distance through the sample. Pfeiffer et al. [23] have shown that the final absorption spectrum can vary significantly depending on the length of the gas sample, because regions of the sample where the lineshape is Lorentzian alternate with regions where the lineshape is Fano-like due to the phase shift induced by the NIR pulse. The final measured spectrum then depends sensitively on the length of the gas sample.

These calculations and experiments have reinforced the importance of accurate consideration of experimental conditions in a transient absorption measurement. An experimental measurement cannot necessarily be adequately modeled by a single-atom calculation alone.

\section{Outlook}

Transient absorption, with the capability to simultaneously achieve high spectral resolution and high temporal resolution, is an important tool to study ultrafast dynamics in molecular systems. The next frontier for attosecond transient absorption is to apply the techniques that are well established for atomic studies to more complicated molecular systems. The absorption features will not be as strong or discrete, and excellent spectral resolution will be necessary to resolve closely-spaced vibrational and even rotational levels.

The ability to monitor individual absorption features allows the lifetimes of shortlived molecular states to be tracked in real time. Extracting a lifetime directly from an absorption lineshape in the densely packed absorption spectrum of a complicated molecular system is difficult. Other processes such as vibrational broadening or ligand field splittings also contribute to the observed lineshape, so any lifetime determination from static absorption spectra alone is error-prone. Initial experiments could measure the lifetimes of states with resonances well-separated from other molecular transitions, such as the absorption features corresponding to core-level excitations of a heavy atom in a small molecule. The measured lifetimes could then be compared to those predicted from static absorption spectra in order to investigate whether broadening effects are accurately accounted for.

The idea of measuring lifetimes in molecular systems seems simple, but the realistic situation in many molecular systems is likely to be much more complex. Many short-lived states manifest in absorption spectra as broad features buried underneath sharper absorption lines corresponding to Rydberg series. Tracking the decays of these broad features and disentangling their time-dependent behavior from the behavior of the overlapping features may still be difficult. However, tracking states through their contribution to a beating wavepacket may allow individual lifetimes to be determined more easily. If some of the members of an initially excited wavepacket can decay, the changing composition of the wavepacket as a function of time could be measured by monitoring any of the features in the initially excited wavepacket. Long-lived states in the wavepacket would not show a decay in the absorption feature, but the amplitude of 
the beating could decrease as the other states in the wavepacket decay. This decay would differ from the state lifetime by a factor of two, as in the direct lifetime measurements.

Wavepacket excitation in an atom merely results in oscillation of the excited population between different electronic states. However, in a molecular system, wavepackets can be composed of excitations to molecular orbitals localized at different sites in the molecule. Then, the wavepacket oscillation manifests as motion of the charge between different atoms or bonds. This has been shown theoretically by the groups of Cederbaum (as in Refs. [42,43]) and Remacle and Levine (as in Refs. [44,45]) for many small molecular systems. In these calculations, an electron is removed from the highest occupied molecular orbital and the resulting wavepacket oscillates on a few-fs or faster timescale. This ionization could be achieved by strong field ionization using the intense NIR pulse, but the initial wavepacket could also be excited using an attosecond XUV pulse and then the resulting wavepacket motion could be probed with a perturbing timedelayed NIR pulse.

Attosecond transient absorption experiments have so far been performed only with a probing pulse centered around $800 \mathrm{~nm}$, with a broadband spectrum spanning the visible and near-infrared regions of the spectrum. A straightforward expansion of the technique would be the use of a varying wavelength probe pulse. The subcycle fringes in the transient absorption spectra due to interference between a direct and an indirect pathway were shown to depend sensitively on the position of an intermediate state in the indirect pathway, as discussed in section 4.1. A first experiment measuring the subcycle fringes in helium could confirm the expected theoretical behavior of the fringes as the wavelength of the probe pulse is scanned.

Varying the probe pulse wavelength is an experimentally feasible way to observe the smooth transition from a LIS to Autler-Townes splitting (or vice versa) as shown in Figure 4. Also, the energies at which the LISs are observed in the transient absorption spectrum depend on the energies of the dark states in the system and the NIR photon energy. If the dark state energies are known, the LIS features could be moved merely by tuning the wavelength to the appropriate energy. Alternately, varying the probe wavelength and noting when Autler-Townes splitting is observed rather than LISs could be used to locate dark states, which would identify the strongest couplings between the one-photon allowed states and nearby dark states.

A variable wavelength probe pulse could also be applied to extract new information from lifetime measurements or observation of wavepacket beating. Different coupling pathways between a decaying state and dark states could be preferentially selected to modify the observed decay. The beating strength of each feature in a complicated wavepacket could also provide information about relative coupling strengths between bright and dark states, as the beating strength would change as the probing pulse wavelength is varied. The ability to change the wavelength of the probe pulse would allow manipulation of a target system in order to study the couplings between specific states.

Another future direction for attosecond transient absorption experiments is experiments with two or more attosecond pulses. The photon flux of attosecond sources has continuously improved since the first attosecond pulses were produced. With the development of higher-power laser systems, splitting an attosecond pulse into two pulses with an experimentally useful number of photons in each pulse will soon be realized. 
Experiments could be performed with only two attosecond pulses, or with two attosecond pulses and a NIR or other ultrashort pulse that perturbs the system. Schemes for threepulse experiments based on spectral interferometry techniques have been previously proposed [7]. Two attosecond pulses separated by some time delay propagate through a medium, and the final measured spectrum displays an interference pattern from those two pulses. If in between the pulses, some change is induced in the medium (such as ionization of a gas-phase sample), the behavior of the system can be probed by monitoring changes in this interference pattern. This technique, dubbed transient dispersion by Pfeifer et al. [7], was initially proposed as a method to measure the index of refraction of the system by comparing the spectral phases of the two attosecond pulses. However, the second attosecond pulse could also be considered as a direct probe of how the induced polarization has been modified by the NIR pulse.

Multiple attosecond pulses could even be used to apply the ideas of twodimensional IR spectroscopy to the XUV regime, as proposed by Mukamel et al. [46]. In 2D IR experiments, a sequence of broadband femtosecond pulses is used to first induce a polarization (or free induction decay, in the language of multidimensional spectroscopy) and then perturb or manipulate this polarization [47]. Cross peaks in the spectrum then reveal the couplings between, for example, vibrational states. These methods could be extended into the XUV energy range to study couplings between electronic states.

\section{Conclusion}

Attosecond transient absorption has proven to be a powerful technique for direct measurement of ultrafast dynamics in atomic systems. New phenomena are observed when the NIR and XUV pulses are overlapped in time, such as light-induced states and subcycle interference fringes. Experiments can also be performed in the counterintuitive regime of time delays where the NIR pulse follows the XUV pulse by a few fs or more, and perturbs the polarization induced by the XUV pulse. Direct measurement of decay lifetimes in the time domain and observation and characterization of quantum beating are just a few examples of the wide applicability of this regime of attosecond transient absorption experiments. In the future, these methods can be extended to investigate ultrafast dynamics in complex molecular systems, which cannot be easily understood from static absorption spectra. Experimental techniques such as varying the wavelength of the probing pulse or generating multiple attosecond pulses to interact with the sample will allow a wider range of atomic and molecular phenomena to be investigated.

\section{Acknowledgements}

This work was supported by the Director, Office of Science, Office of Basic Energy Sciences, and by the Division of Chemical Sciences, Geosciences, and Biosciences of the U.S. Department of Energy at LBNL under Contract No. DE-AC02-05CH11231. A. R. B. acknowledges funding from the National Science Foundation Graduate Research Fellowship Program. S. R. L. acknowledges the support of the Office of Assistant Secretary of Defense for Research and Engineering through a National Security Science and Engineering Faculty Fellowship. 


\section{References}

[1] P. B. Corkum, Phys. Rev. Lett. 71, 1994 (1993).

[2] M. Drescher, M. Hentschel, R. Kienberger, M. Uiberacker, V. Yakovlev, A. Scrinzi, T. Westerwalbesloh, U. Kleineberg, U. Heinzmann, and F. Krausz, Nature 419, 803 (2002).

[3] M. Schultze, M. Fiess, N. Karpowicz, J. Gagnon, M. Korbman, M. Hofstetter, S. Neppl, A. L. Cavalieri, Y. Komninos, T. Mercouris, C. A. Nicolaides, R. Pazourek, S. Nagele, J. Feist, J. Burgdorfer, A. M. Azzeer, R. Ernstorfer, R. Kienberger, U. Kleineberg, E. Goulielmakis, F. Krausz, and V. S. Yakovlev, Science 328, 1658 (2010).

[4] M. Schultze, K. Ramasesha, C. D. Pemmaraju, S. A. Sato, D. Whitmore, A. Gandman, J. S. Prell, L. J. Borja, D. Prendergast, K. Yabana, D. M. Neumark, and S. R. Leone, Science 346, 1348 (2014).

[5] W. T. Pollard and R. A. Mathies, Annu. Rev. Phys. Chem. 43, 497 (1992).

[6] Z.-H. Loh, M. Khalil, R. Correa, R. Santra, C. Buth, and S. Leone, Phys. Rev. Lett. 98, 143601 (2007).

[7] T. Pfeifer, M. J. Abel, P. M. Nagel, A. Jullien, Z.-H. Loh, M. J. Bell, D. M. Neumark, and S. R. Leone, Chem. Phys. Lett. 463, 11 (2008).

[8] E. Goulielmakis, Z.-H. Loh, A. Wirth, R. Santra, N. Rohringer, V. S. Yakovlev, S. Zherebtsov, T. Pfeifer, A. M. Azzeer, M. F. Kling, S. R. Leone, and F. Krausz, Nature 466, 739 (2010).

[9] M. Chini, X. Wang, Y. Cheng, Y. Wu, D. Zhao, D. A. Telnov, S.-I. Chu, and Z. Chang, Sci. Rep. 3, 1105 (2013).

[10] C. Ott, A. Kaldun, P. Raith, K. Meyer, M. Laux, J. Evers, C. H. Keitel, C. H. Greene, and T. Pfeifer, Science 340, 716 (2013).

[11] H. Mashiko, S. Gilbertson, C. Li, S. D. Khan, M. M. Shakya, E. Moon, and Z. Chang, Phys. Rev. Lett. 100, 103906 (2008).

[12] B. Bernhardt, A. R. Beck, X. Li, E. R. Warrick, M. J. Bell, D. J. Haxton, C. W. McCurdy, D. M. Neumark, and S. R. Leone, Phys. Rev. A 89, 023408 (2014).

[13] A. R. Beck, B. Bernhardt, E. R. Warrick, M. Wu, S. Chen, M. Gaarde, K. J. Schafer, D. M. Neumark, and S. R. Leone, New J. Phys. 16, 113016 (2014).

[14] M. Schultze, E. M. Bothschafter, A. Sommer, S. Holzner, W. Schweinberger, M. Fiess, M. Hofstetter, R. Kienberger, V. Apalkov, V. S. Yakovlev, M. I. Stockman, and F. Krausz, Nature 493, 75 (2012).

[15] M. B. Gaarde, C. Buth, J. L. Tate, and K. J. Schafer, Phys Rev A 83, 013419 (2011).

[16] S. Chen, M. J. Bell, A. R. Beck, H. Mashiko, M. Wu, A. N. Pfeiffer, M. B. Gaarde, D. M. Neumark, S. R. Leone, and K. J. Schafer, Phys. Rev. A 86, 063408 (2012).

[17] S. H. Autler and C. H. Townes, Phys. Rev. 100, 703 (1955).

[18] C. Buth and R. Santra, Phys. Rev. A 78, 043409 (2008).

[19] M. Tarana and C. H. Greene, Phys. Rev. A 85, 013411 (2012).

[20] S. E. Harris, J. E. Field, and A. Imamoğlu, Phys. Rev. Lett. 64, 1107 (1990).

[21] Z.-H. Loh, C. H. Greene, and S. R. Leone, Chem. Phys. 350, 7 (2008).

[22] P. Ranitovic, X. M. Tong, C. W. Hogle, X. Zhou, Y. Liu, N. Toshima, M. M. Murnane, and H. C. Kapteyn, Phys. Rev. Lett. 106, 193008 (2011). 
[23] M. J. Bell, A. R. Beck, H. Mashiko, D. M. Neumark, and S. R. Leone, J. Mod. Opt. 60, 1506 (2013).

[24] S. Chen, M. Wu, M. B. Gaarde, and K. J. Schafer, Phys. Rev. A 88, 033409 (2013).

[25] S. Pabst, A. Sytcheva, A. Moulet, A. Wirth, E. Goulielmakis, and R. Santra, Phys. Rev. A 86, 063411 (2012).

[26] W.-C. Chu and C. Lin, Phys. Rev. A 87, 013415 (2013).

[27] S. Chen, M. Wu, M. B. Gaarde, and K. J. Schafer, Phys. Rev. A 87, 033408 (2013).

[28] X. Wang, M. Chini, Y. Cheng, Y. Wu, X.-M. Tong, and Z. Chang, Phys. Rev. A 87, 063413 (2013).

[29] A. N. Pfeiffer and S. R. Leone, Phys. Rev. A 85, 053422 (2012).

[30] M. Wu, S. Chen, M. B. Gaarde, and K. J. Schafer, Phys. Rev. A 88, 043416 (2013).

[31] M.-F. Lin, A. N. Pfeiffer, D. M. Neumark, S. R. Leone, and O. Gessner, J. Chem. Phys. 137, 244305 (2012).

[32] H. Wang, M. Chini, S. Chen, C.-H. Zhang, F. He, Y. Cheng, Y. Wu, U. Thumm, and Z. Chang, Phys. Rev. Lett. 105, 143002 (2010).

[33] D. L. Ederer, Phys. Rev. A 4, 2263 (1971).

[34] C. H. Brito-Cruz, J. P. Gordon, P. C. Becker, R. L. Fork, and C. V. Shank, IEEE J. Quantum Electron. 24, 261 (1988).

[35] M. Lindberg and S. W. Koch, Phys Rev B 38, 7607 (1988).

[36] X. Li, B. Bernhardt, A. R. Beck, E. R. Warrick, A. N. Pfeiffer, M. J. Bell, D. J. Haxton, C. W. McCurdy, D. M. Neumark, and S. R. Leone, (in preparation).

[37] M. Chini, X. Wang, Y. Cheng, and Z. Chang, J. Phys. B At. Mol. Opt. Phys. 47, 124009 (2014).

[38] C. Ott, A. Kaldun, L. Argenti, P. Raith, K. Meyer, M. Laux, Y. Zhang, A.

Blättermann, S. Hagstotz, T. Ding, R. Heck, J. Madroñero, F. Martín, and T. Pfeifer, Nature 516, 374 (2014).

[39] R. Santra, V. S. Yakovlev, T. Pfeifer, and Z.-H. Loh, Phys. Rev. A 83, 033405 (2011).

[40] M. D. Crisp, Phys. Rev. A 1, 1604 (1970).

[41] D. Strasser, T. Pfeifer, B. J. Hom, A. M. Muller, J. Plenge, and S. R. Leone, Phys. Rev. A 73, 021805(R) (2006).

[42] H. Hennig, J. Breidbach, and L. S. Cederbaum, J. Phys. Chem. A 109, 409 (2005).

[43] S. Lünnemann, A. I. Kuleff, and L. S. Cederbaum, Chem. Phys. Lett. 450, 232 (2008).

[44] B. Mignolet, R. D. Levine, and F. Remacle, J. Phys. B At. Mol. Opt. Phys. 47, 124011 (2014).

[45] F. Remacle and R. D. Levine, Z. Für Phys. Chem. 221, 647 (2007).

[46] S. Mukamel, D. Healion, Y. Zhang, and J. D. Biggs, Annu. Rev. Phys. Chem. 64, 101 (2013).

[47] D. M. Jonas, Annu. Rev. Phys. Chem. 54, 425 (2003). 
Author biographies:

Annelise Beck received her S.B. in Chemistry and Physics from the Massachusetts Institute of Technology in 2009 and her Ph.D. in Physical Chemistry from the University of California, Berkeley in 2014 under the supervision of Professor Stephen R. Leone and Professor Daniel M. Neumark. She is currently a Senior Process Engineer at Intel.

Daniel M. Neumark is Professor of Chemistry at the University of California, Berkeley. His research interests encompass fundamental reaction dynamics, electron solvation dynamics in clusters and liquid jets, ultrafast $\mathrm{x}$-ray science, and electron interactions with nucleic acid constituents. He has developed novel negative ion photodetachment techniques to address these topics. His awards include the American Chemical Society Nobel Laureate Signature Award (with Martin Zanni) (2000), the Bomem-Michelson Award (2001), theWilliam F. Meggers Award (2005), the Irving Langmuir Award (2008), Herschbach Medal (2009), the Herbert P. Broida Prize (2013), and the Royal Society of Chemistry Award in Chemical Dynamics (2013).

Stephen R. Leone is Professor of Chemistry and Physics at the University of California, Berkeley. He held previous positions at USC (1974-1976), NIST, JILA, and the University of Colorado (1976-2002). His awards include the American Chemical Society Peter Debye Award (2005), Polanyi Medal of the Gas Kinetics Division of the Royal Society of Chemistry, UK (2010), Irving Langmuir Prize in Chemical Physics (2011), American Physical Society, member of the National Academy of Sciences (1995), and fellow of the American Academy of Arts and Sciences (2000). His research interests are in ultrafast and attosecond processes, chemical dynamics, and nanomaterials. 


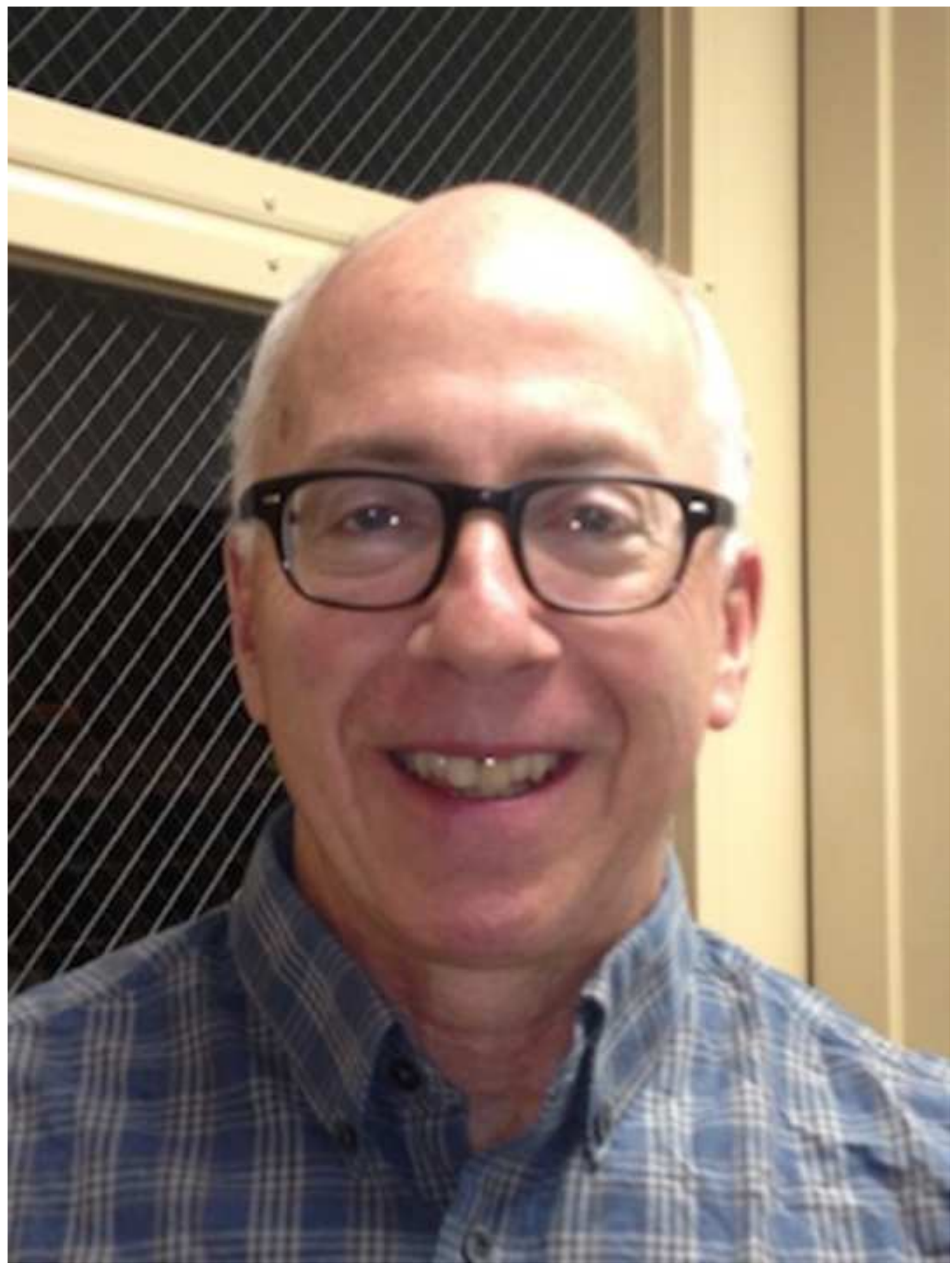


Author Photo SRL

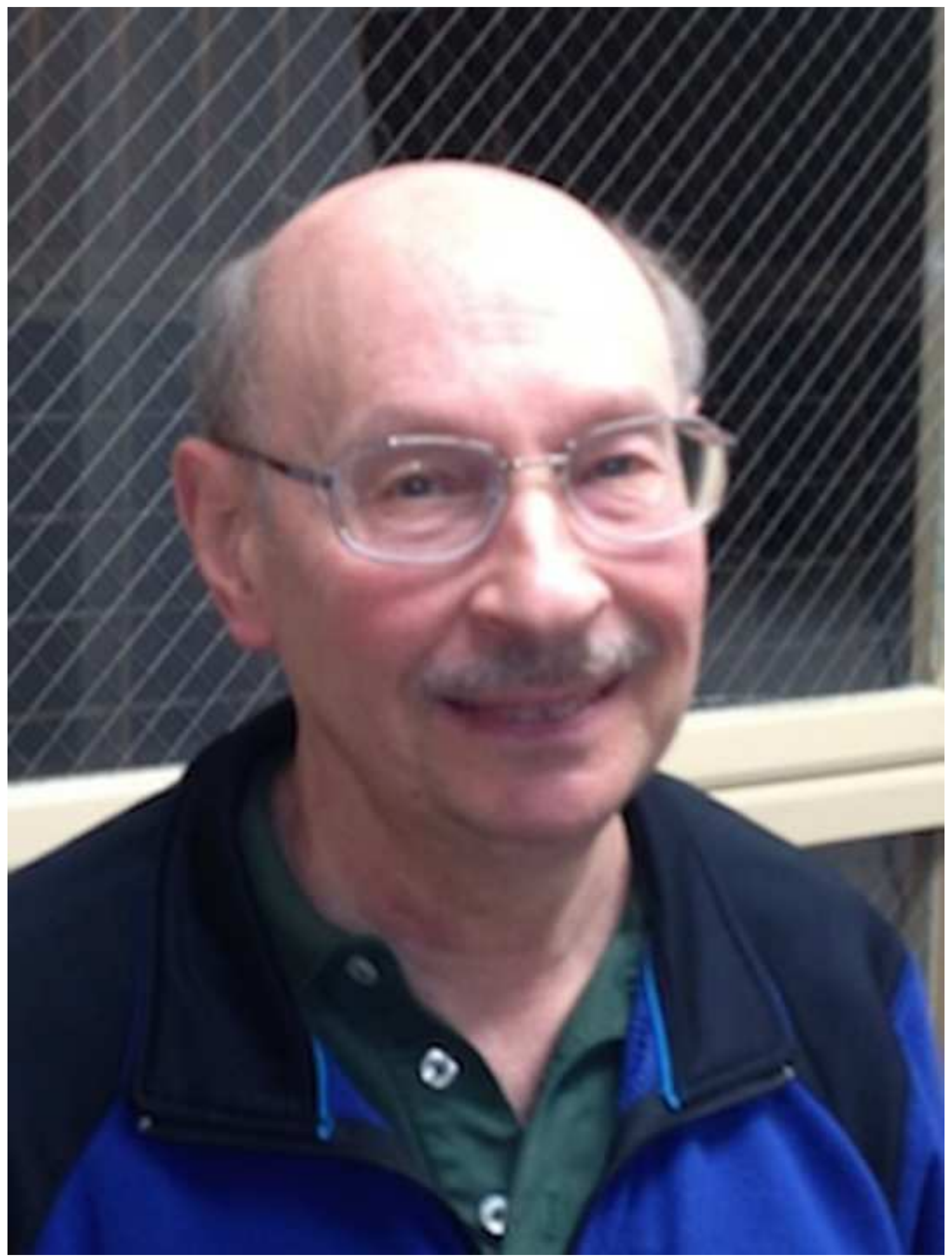




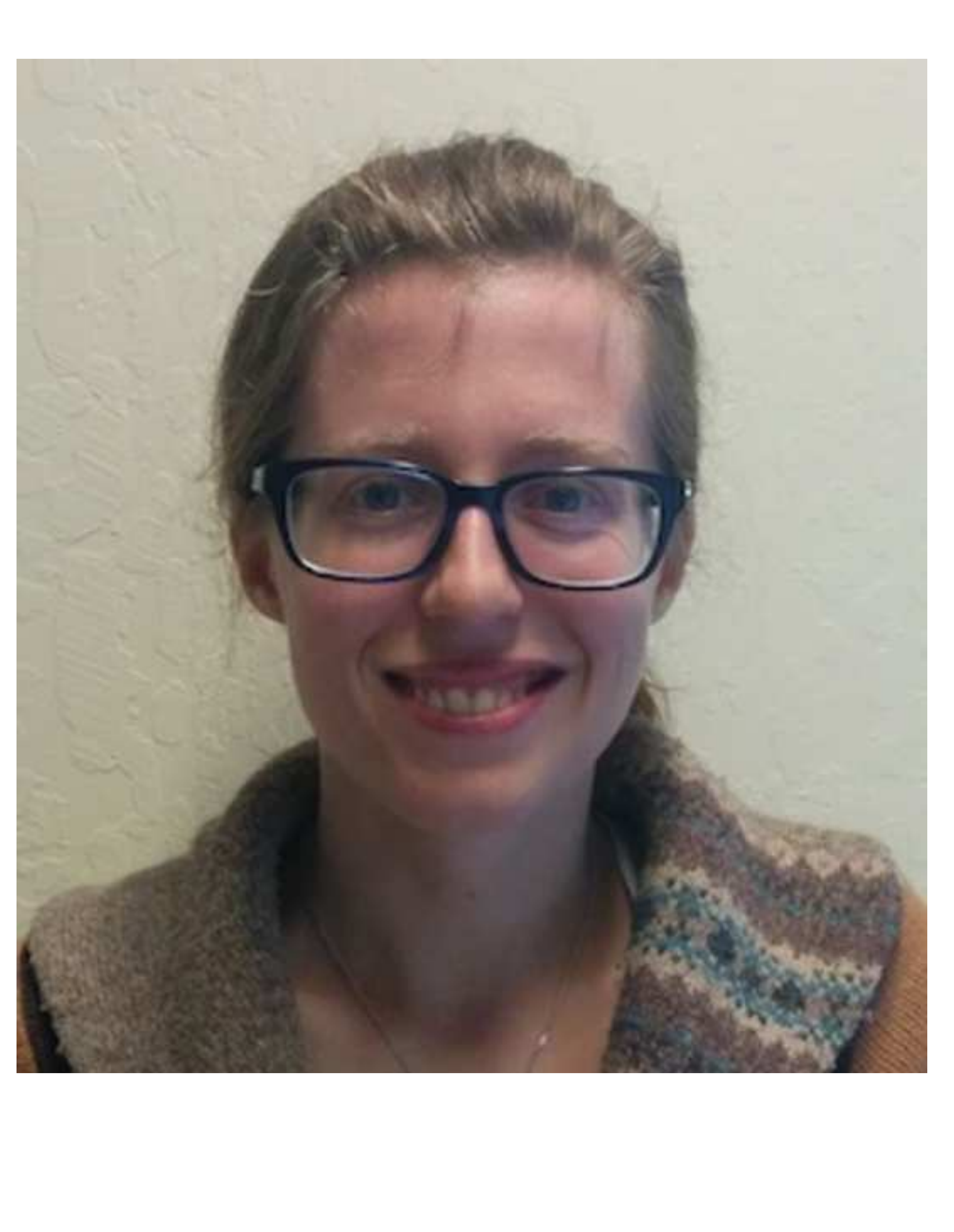

\section{Author Photo ARB}
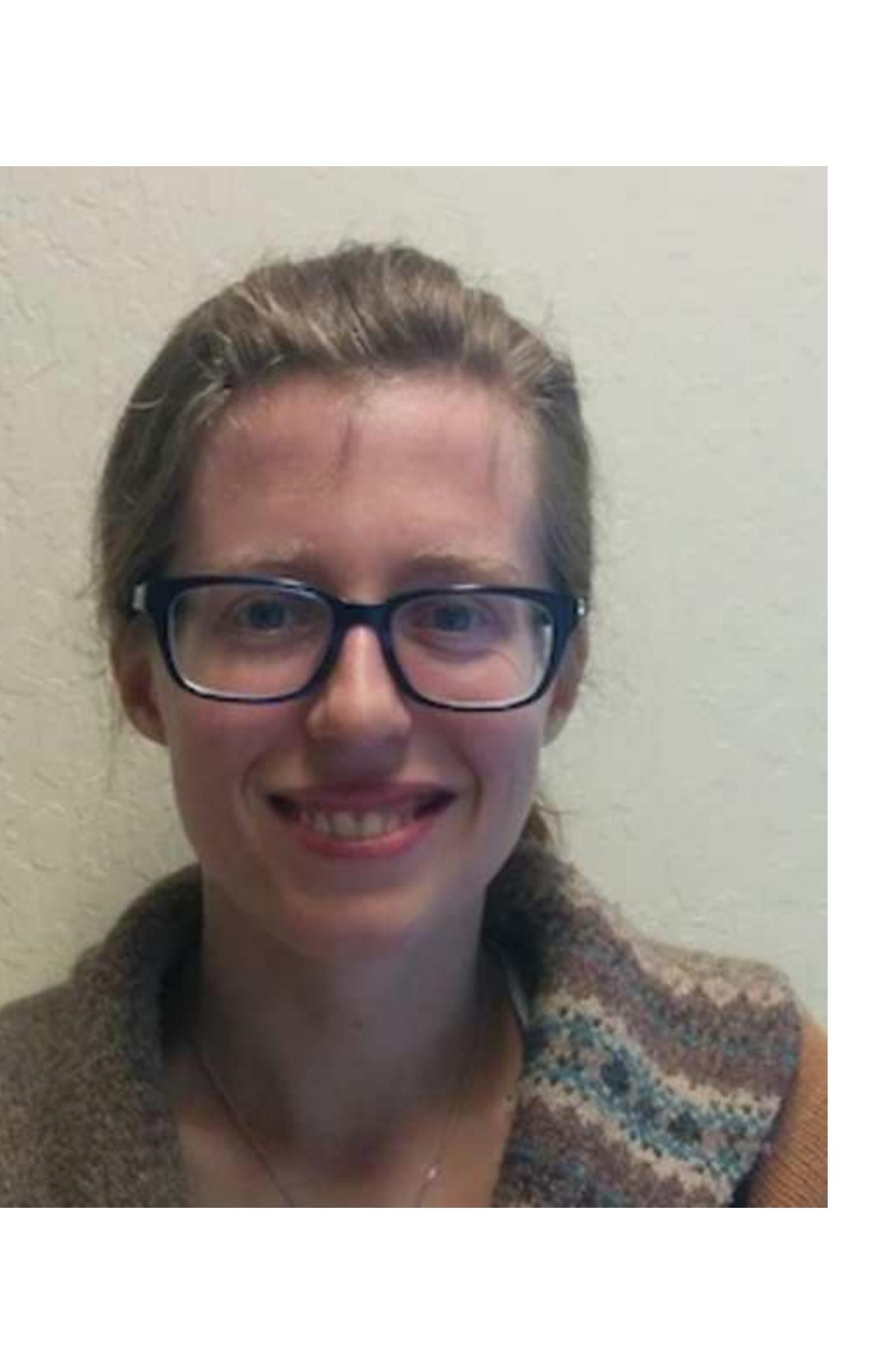

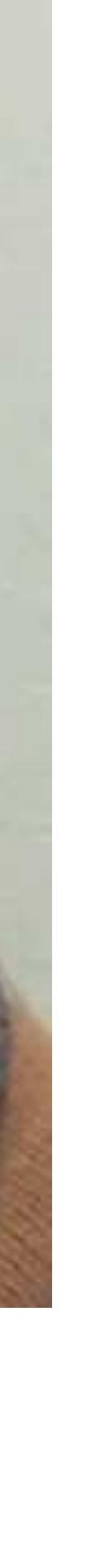

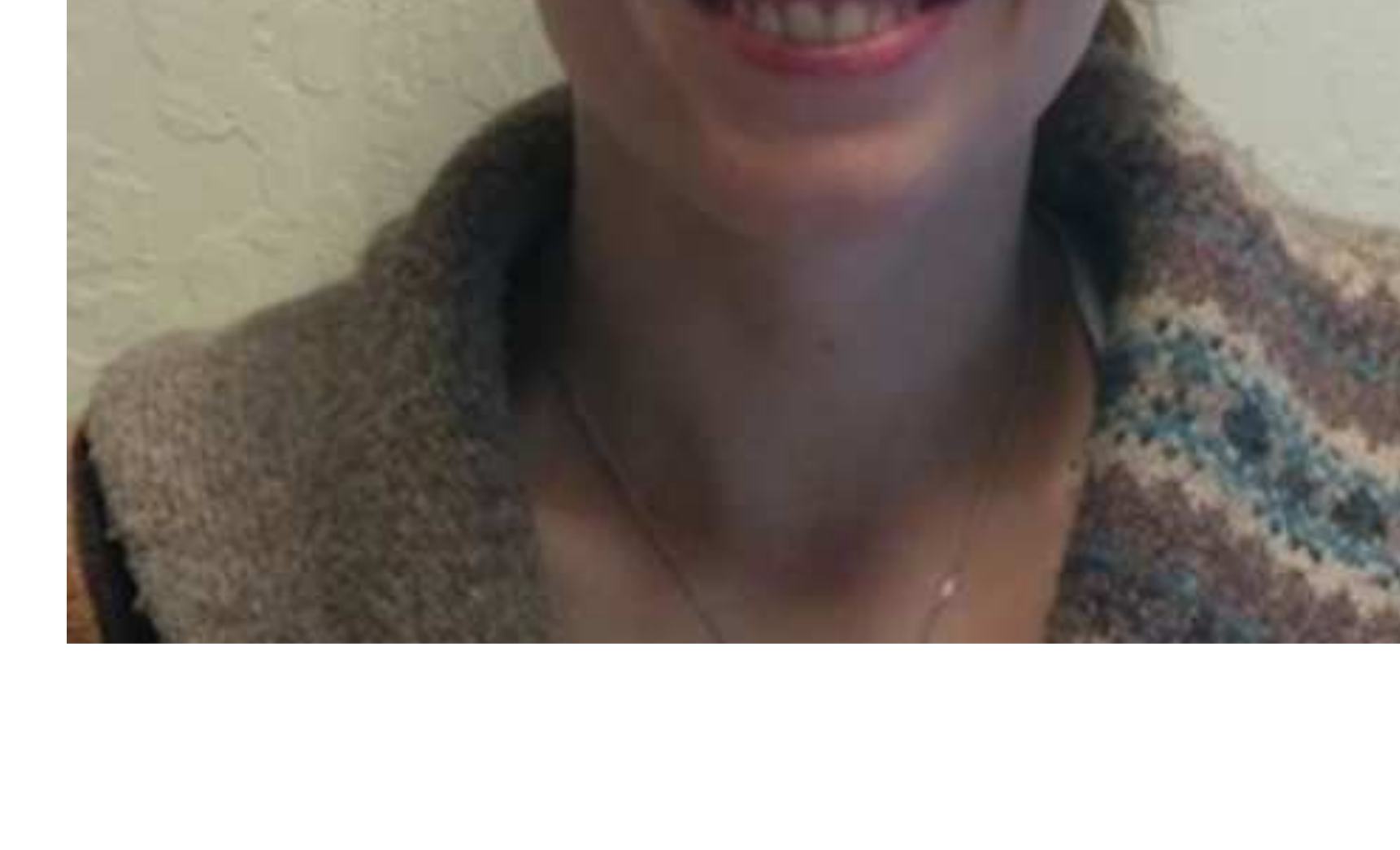

